\title{
The Multistage Homotopy Perturbation Method for Solving Chaotic and Hyperchaotic Lü System
}

\author{
M. S. H. Chowdhury, ${ }^{1}$ Nur Isnida Razali, ${ }^{2}$ Waqar Asrar, ${ }^{2}$ and M. M. Rahman ${ }^{3}$ \\ ${ }^{1}$ Department of Science in Engineering, Faculty of Engineering, International Islamic University Malaysia, Jalan Gombak, \\ 53100 Kuala Lumpur, Malaysia \\ ${ }^{2}$ Department of Mechanical Engineering, Faculty of Engineering, International Islamic University Malaysia, Jalan Gombak, \\ 53100 Kuala Lumpur, Malaysia \\ ${ }^{3}$ Department of Physics, Faculty of Science, Universiti Putra Malaysia, 43400 Selangor, Malaysia
}

Correspondence should be addressed to M. S. H. Chowdhury; sazzadbd@iium.edu.my

Received 2 June 2014; Revised 20 August 2014; Accepted 16 September 2014

Academic Editor: Saeed Islam

Copyright (C) 2015 M. S. H. Chowdhury et al. This is an open access article distributed under the Creative Commons Attribution License, which permits unrestricted use, distribution, and reproduction in any medium, provided the original work is properly cited.

\begin{abstract}
The multistage homotopy-perturbation method (MHPM) is applied to the nonlinear chaotic and hyperchaotic Lü systems. MHPM is a technique adapted from the standard homotopy-perturbation method (HPM) where the HPM is treated as an algorithm in a sequence of time intervals. To ensure the precision of the technique applied in this work, the results are compared with a fourthorder Runge-Kutta method and the standard HPM. The results show that the MHPM is an efficient and powerful technique in solving both chaotic and hyperchaotic systems.
\end{abstract}

\section{Introduction}

The phenomenon of chaos exists in many nonlinear systems including circuits, mechanical devices, population growth, fluid dynamics, and weather. Chaotic systems are sensitive to the changes in initial conditions and have unpredictable behaviour. The first chaotic system, known as the Lorenz system was discovered in 1963 by Lorenz while solving for equations describing atmospheric flows [1]. Several other chaotic systems have been found including Genesio and Tesi [2], Chen and Ueta [3], and Lü and Chen [4]. Chaotic systems have unpredictable, inconsistent behaviour [5]. Hyperchaotic systems were first reported by Rossler in 1979 [6]. Such systems have more than one positive Lyapunov exponent which indicates that the chaotic dynamics of the system spans more than one direction leading to more complex attractors [7]. Since hyperchaotic systems have multiple positive Lyapunov exponents hence they are more difficult to predict as compared to a chaotic system which has only one positive Lyapunov exponent. Based on their dynamical response, many hyperchaotic systems have been developed including the Li et al. [8], Chen et al. [9], and Jia [10] hyperchaotic systems.

In this paper, we attempt to find approximate solutions of the following Lü chaotic systems (1)-(2):

$$
\begin{aligned}
& \dot{x}=a(y-x), \\
& \dot{y}=-x z+c y, \\
& \dot{z}=x y-b z,
\end{aligned}
$$

where $x, y$, and $z$ are state variables and $a, b$, and $c$ are positive parameters. This system exhibits chaotic behaviour when $a=$ $36, b=3$, and $c=20$. According to a mathematical sense defined by Vaněček and Čelikovské, this system represents the transition between the Lorenz system and the Chen system $[11,12]$. The system of (1) is later referred to as the Lü system [13]. Some detailed investigations on the Lü system (1) can be found in [14] which is a numerical study of their 
dynamical behaviour. Next, the hyperchaotic Lü system [15] is described by

$$
\begin{aligned}
\dot{x} & =a(y-x)+w, \\
\dot{y} & =-x z+c y, \\
\dot{z} & =x y-b z, \\
\dot{w} & =x z+r w,
\end{aligned}
$$

where $x, y, z$, and $w$ are state variables and $a, b, c$, and $r$ are real constants. When $a=36, b=3, c=20$, and $-0.35<$ $r \leq 1.3$, the system of (2) has a hyperchaotic attractor. Most of the chaotic and hyperchaotic systems do not have exact solutions. Thus, a numerical technique has to be used to find approximate solutions of chaotic and hyperchaotic systems. Some classes of dynamical systems have been solved by the Adomian decomposition method (ADM) [16-18] and by the Variational iteration method (VIM) $[19,20]$. In particular, the chaotic Genesio system was solved by Goh et al. via VIM [21]. Hashim et al. and Al-Sawalha et al. [22, 23] solved the Lorenz and hyperchaotic Rossler system by ADM.

The HPM was first developed and improved by He in 1998 [24-29]. Constructing an appropriate homotopy equation and choosing a correct initial guess are the two vital steps in application of the homotopy perturbation method. He [30] reviewed recent development of some new asymptotic methods for the solitary solutions of nonlinear differential and fractional differential equations with some new ideas. This paper also suggests an alternative approach for construction of the homotopy equation with an auxiliary term. The HPM has been successfully applied to solve variety of linear and nonlinear problems in [31-36]. Recently, the application of HPM was extended to chaotic Genesio system [37], heat transfer analysis on the Hiemenz flow of a nonNewtonian fluid [38], long porous slider problem [39], and nonlinear boundary value problems of fractional order [40]. Very recently, Chowdhury et al. [41] introduced modified HPM to solve differential and integral equations.

To the best of our knowledge, the standard HPM does not work accurately in solving chaotic and hyperchaotic systems and stiff systems for long time spans. Finding accurate and efficient techniques to overcome the limitation of the HPM has long been an active research undertaking. In [42], the optimal homotopy perturbation method (OHPM) was applied to find the solutions for a nonconservative dynamical system of a rotating electrical machine. Very recently in [43], Olvera and Elías-Zúñiga have introduced the enhanced multistage homotopy perturbation method (EMHPM) approach to solve nonlinear dynamical systems.

The multistage HPM (MPHM) is a powerful technique to get more reliable and efficient approximate solutions. It is an improvement over the standard HPM. Recently, it (the MHPM) was successfully applied to the chaotic Chen system, Lorenz system, a class of ODEs, a nonlinear biochemical reactor model, and chaotic Rössler system by Chowdhury and Hashim [44], Chowdhury et al. [45], Hashim and Chowdhury [46], Hashim et al. [47], and Chowdhury et al. [48], respectively.
In the literature survey, we observe that very little research work has been done on the MHPM. In this paper, the success of the MHPM motivates us to extend the application of analytic homotopy perturbation method to solve chaotic and hyperchaotic Lü systems. The accuracy of MHPM in solving chaotic and hyperchaotic systems is investigated by providing one example of a chaotic system and three examples of hyperchaotic systems. The solutions obtained by MHPM are compared with the solutions obtained by the standard HPM and fourth-order Runge-Kutta techniques.

\section{Description of the Solution Procedure}

Consider a general system of first-order ODEs:

$$
\begin{gathered}
\frac{d s_{1}}{d t}+g_{1}\left(t, s_{1}, s_{2}, \ldots, s_{m}\right)=f_{1}(t), \\
\frac{d s_{2}}{d t}+g_{2}\left(t, s_{1}, s_{2}, \ldots, s_{m}\right)=f_{2}(t), \\
\vdots \\
\frac{d s_{m}}{d t}+g_{m}\left(t, s_{1}, s_{2}, \ldots, s_{m}\right)=f_{m}(t),
\end{gathered}
$$

subject to the initial conditions

$$
\begin{aligned}
& s_{1}\left(t_{0}\right)=c_{1}, \\
& s_{2}\left(t_{0}\right)=c_{2}, \ldots, s_{m}\left(t_{0}\right)=c_{m} .
\end{aligned}
$$

First we write the system (2) in operator form

$$
\begin{gathered}
L\left(s_{1}\right)+N_{1}\left(s_{1}, s_{2}, \ldots, s_{m}\right)-f_{1}=0, \\
L\left(s_{2}\right)+N_{2}\left(s_{1}, s_{2}, \ldots, s_{m}\right)-f_{2}=0, \\
\vdots \\
L\left(s_{m}\right)+N_{m}\left(s_{1}, s_{2}, \ldots, s_{m}\right)-f_{m}=0,
\end{gathered}
$$

subject to the initial conditions (4), where $L=d / d t$ is a linear operator and $N_{1}, N_{2}, \ldots, N_{m}$ are the nonlinear operators. In the next section, we will provide the solution for (5) based on HPM and MHPM.

2.1. Solution by HPM. According to HPM, we construct the homotopy for (5) which satisfies the following relations:

$$
\begin{gathered}
L\left(s_{1}\right)-L\left(v_{1}\right)+p L\left(v_{1}\right)+p\left[N_{1}\left(s_{1}, s_{2}, \ldots, s_{m}\right)-f_{1}\right]=0, \\
L\left(s_{2}\right)-L\left(v_{2}\right)+p L\left(v_{2}\right)+p\left[N_{2}\left(s_{1}, s_{2}, \ldots, s_{m}\right)-f_{2}\right]=0, \\
\vdots \\
L\left(s_{m}\right)-L\left(v_{m}\right)+p L\left(v_{m}\right)+p\left[N_{m}\left(s_{1}, s_{2}, \ldots, s_{m}\right)-f_{m}\right]=0,
\end{gathered}
$$

where $p \in[0,1]$ is an embedding parameter and $v_{1}, v_{2}, \ldots, v_{m}$ are initial approximations satisfying the given conditions. It 
is obvious that when the perturbation parameter $p=0,(6)$ reduce to a linear system and when $p=1$, we get the original nonlinear system. Let us assume the approximations are as follows:

$$
\begin{aligned}
s_{1}(t) & =s_{1,0}(t)+p s_{1,1}(t)+p^{2} s_{1,2}(t)+p^{3} s_{1,3}(t)+\cdots \\
s_{2}(t) & =s_{2,0}(t)+p s_{2,1}(t)+p^{2} s_{2,2}(t)+p^{3} s_{2,3}(t)+\cdots \\
& \vdots \\
s_{m}(t) & =s_{m, 0}(t)+p s_{m, 1}(t)+p^{2} s_{m, 2}(t)+p^{3} s_{m, 3}(t)+\cdots, \\
s_{1,0}(t) & =v_{1}(t)=s_{1}\left(t_{0}\right)=c_{1}, \\
s_{2,0}(t) & =v_{2}(t)=s_{2}\left(t_{0}\right)=c_{2}, \\
& \vdots \\
s_{m, 0}(t) & =v_{m}(t)=s_{m}\left(t_{0}\right)=c_{m},
\end{aligned}
$$

where $s_{i, j}(i=1,2, \ldots, m ; j=1,2, \ldots)$ are functions yet to be determined. Substituting (7) into (6) and arranging the coefficient of like powers of $p$ we obtain

$$
\begin{aligned}
& L\left(s_{1,1}\right)+L v_{1}+N_{1}\left(s_{1,0}, s_{2,0}, \ldots, s_{m, 0}\right)-f_{1}=0, \\
& s_{1,1}\left(t_{0}\right)=0, \\
& L\left(s_{2,1}\right)+L v_{2}+N_{2}\left(s_{1,0}, s_{2,0}, \ldots, s_{m, 0}\right)-f_{2}=0, \\
& s_{2,1}\left(t_{0}\right)=0 \\
& \vdots \\
& L\left(s_{m, 1}\right)+L v_{m}+N_{m}\left(s_{1,0}, s_{2,0}, \ldots, s_{m, 0}\right)-f_{m}=0, \\
& s_{m, 1}\left(t_{0}\right)=0, \\
& L\left(s_{1,2}\right)+N_{1}\left(s_{1,1}, s_{2,1}, \ldots, s_{m, 1}\right)=0, \\
& s_{1,2}\left(t_{0}\right)=0, \\
& L\left(s_{2,2}\right)+N_{2}\left(s_{1,1}, s_{2,1}, \ldots, s_{m, 1}\right)=0, \\
& s_{2,2}\left(t_{0}\right)=0, \\
& L\left(s_{m, 2}\right)+N_{m}\left(s_{1,1}, s_{2,1}, \ldots, s_{m, 1}\right)=0, \\
& s_{m, 2}\left(t_{0}\right)=0,
\end{aligned}
$$

and so forth. We solve the above systems of equations for the unknown $s_{i, j}(i=1,2, \ldots, m ; j=1,2, \ldots)$ by applying the inverse operator

$$
L^{-1}(\cdot)=\int_{t_{0}}^{t}(\cdot) d t .
$$

Therefore, according to HPM the $n$-term approximations for the solutions of (5) can be expressed as

$$
\begin{gathered}
\phi_{1, n}(t)=s_{1}(t)=\lim _{p \rightarrow 1} s_{1}(t)=\sum_{k=0}^{n-1} s_{1, k}(t), \\
\phi_{2, n}(t)=s_{2}(t)=\lim _{p \rightarrow 1} s_{2}(t)=\sum_{k=0}^{n-1} s_{2, k}(t), \\
\vdots \\
\phi_{m, n}(t)=s_{m}(t)=\lim _{p \rightarrow 1} s_{m}(t)=\sum_{k=0}^{n-1} s_{m, k}(t) .
\end{gathered}
$$

The solution obtained by HPM is not valid for large $t$. A simple way of ensuring validity of the approximations for large $t$ is to treat the algorithm of HPM in a sequence of intervals choosing the initial approximations as

$$
\begin{gathered}
s_{1,0}(t)=v_{1}(t)=s_{1}\left(t^{*}\right)=c_{1}^{*} \\
s_{2,0}(t)=v_{2}(t)=s_{2}\left(t^{*}\right)=c_{2}^{*} \\
\vdots \\
s_{m, 0}(t)=v_{m}(t)=s_{m}\left(t^{*}\right)=c_{m}^{*},
\end{gathered}
$$

where $t^{*}$ is the left-end point of each subinterval. Then solve (8) for the unknowns $s_{i, j}(i=1,2, \ldots, m ; j=1,2, \ldots)$ by applying the inverse linear operator

$$
L^{-1}(\cdot)=\int_{t^{*}}^{t}(\cdot) d t .
$$

In order to carry out the iterations in every subinterval of equal length $\Delta t,\left[0, t_{1}\right),\left[t_{1}, t_{2}\right) \cdots\left[t_{j-1}, t\right)$, we need to know the values of

$$
\begin{gathered}
s_{1,0}^{*}(t)=s_{1}\left(t^{*}\right) \\
s_{2,0}^{*}(t)=s_{2}\left(t^{*}\right), \ldots, s_{m, 0}^{*}(t)=s_{m}\left(t^{*}\right) .
\end{gathered}
$$

But in general, we do not have this information except at the initial point $t^{*}=t_{0}$. A simple method to obtain the necessary values could be by means of the previous $n$ terms of approximations $\phi_{1, n}, \phi_{2, n}, \ldots, \phi_{m, n}$ of the preceding subinterval given by (10); that is,

$$
\begin{aligned}
& s_{1,0}^{*} \approx \phi_{1, n}\left(t^{*}\right), \\
& s_{2,0}^{*} \approx \phi_{2, n}\left(t^{*}\right), \ldots, s_{m, 0}^{*} \approx \phi_{m, n}\left(t^{*}\right) .
\end{aligned}
$$

\section{Applications}

3.1. Chaotic Lü System. The chaotic Lü system (1) subject to initial conditions is

$$
x\left(t_{0}\right)=c_{1}, \quad y\left(t_{0}\right)=c_{2}, \quad z\left(t_{0}\right)=c_{3} .
$$


TABLE 1: Determination of accuracy of RK4 for chaotic Lü system.

\begin{tabular}{|c|c|c|c|c|c|c|}
\hline \multirow{2}{*}{$t$} & \multicolumn{3}{|c|}{$\Delta=\left|\mathrm{RK} 4_{0.01}-\mathrm{RK} 4_{0.001}\right|$} & \multicolumn{3}{|c|}{$\Delta=\left|\mathrm{RK} 4_{0.001}-\mathrm{RK} 4_{0.0001}\right|$} \\
\hline & $\Delta x$ & $\Delta y$ & $\Delta z$ & $\Delta x$ & $\Delta y$ & $\Delta z$ \\
\hline 0.5 & 0.001434 & 0.001185 & 0.0003733 & $1.005 E-07$ & $7.881 E-08$ & $4.693 E-09$ \\
\hline 1.0 & 0.000913 & 0.001317 & 0.001694 & $3.371 E-08$ & $6.750 E-08$ & $1.256 E-07$ \\
\hline 1.5 & 0.003576 & 0.003748 & 0.0007725 & $2.338 E-07$ & $2.362 E-07$ & $1.797 E-08$ \\
\hline 2.0 & 0.001033 & 0.004104 & 0.01421 & $1.034 E-07$ & $2.283 E-07$ & $8.886 E-07$ \\
\hline 2.5 & 0.0212 & 0.01711 & 0.0182 & $1.300 E-06$ & $1.048 E-06$ & $1.121 E-06$ \\
\hline 3.0 & 0.03132 & 0.02575 & 0.005015 & $1.941 E-06$ & $1.589 E-06$ & $3.214 E-07$ \\
\hline 3.5 & 0.0189 & 0.02265 & 0.003675 & $1.146 E-06$ & $1.376 E-06$ & $2.293 E-07$ \\
\hline 4.0 & 0.2626 & 0.1676 & 0.1903 & $1.563 E-05$ & $9.836 E-06$ & $1.147 E-05$ \\
\hline 4.5 & 0.1756 & 0.2853 & 0.09396 & $1.094 E-05$ & $1.735 E-05$ & $5.097 E-06$ \\
\hline 5.0 & 1.166 & 0.6354 & 2.571 & $5.726 E-05$ & $1.724 E-05$ & 0.0001507 \\
\hline
\end{tabular}

TABLE 2: Determination of accuracy of RK4 for hyperchaotic Lü system for Case 1.

\begin{tabular}{|c|c|c|c|c|c|c|c|c|}
\hline \multirow{2}{*}{$t$} & \multicolumn{4}{|c|}{$\Delta=\left|\mathrm{RK} 4_{0.01}-\mathrm{RK} 4_{0.001}\right|$} & \multicolumn{4}{|c|}{$\Delta=\left|\mathrm{RK} 4_{0.001}-\mathrm{RK} 4_{0.0001}\right|$} \\
\hline & $\Delta x$ & $\Delta y$ & $\Delta z$ & $\Delta w$ & $\Delta x$ & $\Delta y$ & $\Delta z$ & $\Delta w$ \\
\hline 0.5 & 0.0002375 & 0.0001481 & $3.280 E-05$ & 0.0005867 & $1.955 E-08$ & $1.067 E-08$ & $7.956 E-09$ & $6.043 E-08$ \\
\hline 1.0 & 0.0002405 & 0.0002437 & 0.0001312 & 0.001349 & $2.299 E-09$ & $1.431 E-09$ & $9.089 E-09$ & $1.189 E-07$ \\
\hline 1.5 & 0.001621 & 0.001809 & 0.001383 & 0.001686 & $1.486 E-07$ & $1.683 E-07$ & $9.839 E-08$ & $1.346 E-07$ \\
\hline 2.0 & 0.0005217 & 0.0002725 & 0.002093 & 0.0009465 & $4.048 E-08$ & $2.504 E-08$ & $1.678 E-07$ & $1.720 E-07$ \\
\hline 2.5 & 0.01017 & 0.01622 & 0.0009276 & 0.02225 & $8.147 E-07$ & $1.303 E-06$ & $9.630 E-08$ & $1.632 E-06$ \\
\hline 3.0 & 0.004277 & 0.002561 & 0.00404 & 0.005614 & $3.606 E-07$ & $1.907 E-07$ & $3.559 E-07$ & $1.745 E-07$ \\
\hline 3.5 & 0.003124 & 0.00235 & 0.0008089 & 0.000754 & $2.595 E-07$ & $1.473 E-07$ & $1.673 E-07$ & $3.415 E-07$ \\
\hline 4.0 & 0.003355 & 0.004273 & 0.001246 & 0.002474 & $5.262 E-08$ & $4.854 E-08$ & $1.174 E-07$ & $8.625 E-07$ \\
\hline 4.5 & 0.001265 & 0.001878 & 0.006727 & 0.01775 & $3.623 E-07$ & $4.214 E-07$ & $2.982 E-07$ & $1.357 E-06$ \\
\hline 5.0 & 0.02107 & 0.02556 & 0.01099 & 0.003839 & $1.576 E-06$ & $1.956 E-06$ & $6.829 E-07$ & $2.406 E-07$ \\
\hline
\end{tabular}

According to HPM, we can construct a homotopy which satisfies the following relations:

$$
\begin{aligned}
& \dot{u}_{1}-\dot{x}_{0}+p\left(\dot{x}_{0}-a u_{2}+a u_{1}\right)=0, \\
& \dot{u}_{2}-\dot{y}_{0}+p\left(\dot{y}_{0}+u_{1} u_{3}-c u_{2}\right)=0, \\
& \dot{u}_{3}-\dot{z}_{0}+p\left(\dot{z}_{0}-u_{1} u_{2}+b u_{3}\right)=0 .
\end{aligned}
$$

We take the initial approximations as

$$
\begin{gathered}
u_{1,0}(t)=x_{0}(t)=x\left(t^{*}\right)=c_{1}, \\
u_{2,0}(t)=y_{0}(t)=y\left(t^{*}\right)=c_{2}, \\
u_{3,0}(t)=z_{0}(t)=z\left(t^{*}\right)=c_{3}, \\
u_{1}(t)=u_{1,0}(t)+p u_{1,1}(t)+p^{2} u_{1,2}(t)+p^{3} u_{1,3}(t)+\cdots, \\
u_{2}(t)=u_{2,0}(t)+p u_{2,1}(t)+p^{2} u_{2,2}(t)+p^{3} u_{2,3}(t)+\cdots, \\
u_{3}(t)=u_{3,0}(t)+p u_{3,1}(t)+p^{2} u_{3,2}(t)+p^{3} u_{3,3}(t)+\cdots,
\end{gathered}
$$

where $u_{i, j}(t), i=j=1,2,3, \ldots$ are functions yet to be determined. Substituting (17) into (16) and arranging the terms with the same powers of $p$, we have;

$$
\begin{gathered}
\dot{u}_{1,1}-a u_{2,0}+a u_{1,0}=0, \\
\dot{u}_{1,2}-a u_{2,1}+a u_{1,1}=0, \\
\dot{u}_{1,3}-a u_{2,2}+a u_{1,2}=0, \\
\dot{u}_{2,1}+u_{1,0} u_{3,0}-c u_{2,0}=0, \\
\dot{u}_{2,2}+u_{1,0} u_{3,1}+u_{1,1} u_{3,0}-c u_{2,1}=0, \\
\dot{u}_{2,3}+u_{1,0} u_{3,2}+u_{1,1} u_{3,1}+u_{1,2} u_{3,0}-c u_{2,2}=0, \\
\dot{u}_{3,1}-u_{1,0} u_{2,0}+b u_{3,0}=0, \\
\dot{u}_{3,2}-u_{1,0} u_{2,1}-u_{1,1} u_{2,0}+b u_{3,1}=0, \\
\dot{u}_{3,3}-u_{1,0} u_{2,2}-u_{1,1} u_{2,1}-u_{1,2} u_{2,0}+b u_{3,2}=0 .
\end{gathered}
$$

Solve for the unknowns of the (18) by taking the initial conditions as $u_{i, j}(0)=0, i, j=1,2,3$

$$
\begin{aligned}
& u_{1,1}(t)=\left[a c_{2}-a c_{1}\right]\left(t-t^{*}\right), \\
& u_{2,1}(t)=\left[-c_{1} c_{3}+c c_{2}\right]\left(t-t^{*}\right), \\
& u_{3,1}(t)=\left[c_{1} c_{2}-b c_{3}\right]\left(t-t^{*}\right),
\end{aligned}
$$


TABLE 3: Determination of accuracy of RK4 for hyperchaotic Lü system for Case 2.

\begin{tabular}{|c|c|c|c|c|c|c|c|c|}
\hline \multirow{2}{*}{$t$} & \multicolumn{4}{|c|}{$\Delta=\left|\mathrm{RK} 4_{0.01}-\mathrm{RK} 4_{0.001}\right|$} & \multicolumn{4}{|c|}{$\Delta=\left|\mathrm{RK} 4_{0.001}-\mathrm{RK} 4_{0.0001}\right|$} \\
\hline & $\Delta x$ & $\Delta y$ & $\Delta z$ & $\Delta w$ & $\Delta x$ & $\Delta y$ & $\Delta z$ & $\Delta w$ \\
\hline 0.5 & 0.003484 & 0.002306 & 0.003751 & 0.003101 & $2.402 E-07$ & $1.477 E-07$ & $1.948 E-07$ & $4.361 E-07$ \\
\hline 1.0 & 0.005802 & 0.003364 & 0.009935 & 0.02557 & $3.230 E-07$ & $1.671 E-07$ & $5.436 E-07$ & $1.878 E-06$ \\
\hline 1.5 & 0.01226 & 0.007642 & 0.01498 & 0.012 & $6.005 E-07$ & $2.846 E-07$ & $9.289 E-07$ & $1.075 E-06$ \\
\hline 2.0 & 0.0256 & 0.009117 & 0.06103 & 0.05824 & $1.538 E-06$ & $3.303 E-07$ & $4.159 E-06$ & $3.999 E-06$ \\
\hline 2.5 & 0.02291 & 0.03217 & 0.06284 & 0.1317 & $1.136 E-06$ & $1.669 E-06$ & $3.666 E-06$ & $9.756 E-06$ \\
\hline 3.0 & 0.02907 & 0.03988 & 0.03916 & 0.2637 & $2.159 E-06$ & $2.944 E-06$ & $2.533 E-06$ & $1.780 E-05$ \\
\hline 3.5 & 0.182 & 0.2343 & 0.07347 & 0.1774 & $1.230 E-05$ & $1.588 E-05$ & $4.910 E-06$ & $1.254 E-05$ \\
\hline 4.0 & 0.3564 & 0.4636 & 0.9351 & 3.64 & $3.463 E-05$ & $2.798 E-05$ & $7.285 E-05$ & 0.0002693 \\
\hline 4.5 & 0.4772 & 0.4275 & 0.4257 & 2.468 & $3.844 E-05$ & $3.378 E-05$ & $3.462 E-05$ & 0.0001885 \\
\hline 5.0 & 0.423 & 0.5657 & 0.06116 & 2.691 & $3.208 E-05$ & $4.220 E-05$ & $2.855 E-07$ & 0.0002054 \\
\hline
\end{tabular}

TABLE 4: Determination of accuracy of RK4 for hyperchaotic Lü system for Case 3.

\begin{tabular}{ccccccccc}
\hline$t$ & \multicolumn{3}{c}{$\Delta=\left|\mathrm{RK} 4_{0.01}-\mathrm{RK} 4_{0.001}\right|$} & \multicolumn{3}{c}{$\Delta=\left|\mathrm{RK} 4_{0.001}-\mathrm{RK}_{0.0001}\right|$} \\
& $\Delta x$ & $\Delta y$ & $\Delta z$ & $\Delta w$ & $\Delta x$ & $\Delta y$ & $\Delta z$ \\
\hline 0.5 & 0.002475 & 0.006526 & 0.003265 & 0.01027 & $2.629 E-07$ & $6.162 E-07$ & $2.124 E-07$ & $1.026 E-06$ \\
1.0 & 0.005775 & 0.005532 & 0.003493 & 0.0051 & $4.476 E-07$ & $4.546 E-07$ & $2.483 E-07$ & $6.316 E-07$ \\
1.5 & 0.00111 & 0.004554 & 0.005144 & 0.01193 & $2.166 E-07$ & $4.588 E-07$ & $2.686 E-07$ & $9.033 E-07$ \\
2.0 & 0.005571 & 0.004761 & 0.01041 & 0.008758 & $3.632 E-07$ & $3.035 E-07$ & $7.236 E-07$ & $8.201 E-07$ \\
2.5 & 0.00419 & 0.004521 & 0.000942 & 0.008069 & $3.280 E-07$ & $3.615 E-07$ & $1.068 E-07$ & $7.918 E-07$ \\
3.0 & 0.005047 & 0.002339 & 0.01499 & 0.008466 & $3.620 E-07$ & $1.322 E-07$ & $1.181 E-06$ & $9.253 E-07$ \\
3.5 & 0.001716 & 0.002753 & 0.0001161 & 0.008443 & $1.053 E-07$ & $1.528 E-07$ & $4.942 E-08$ & $8.159 E-07$ \\
4.0 & 0.0009202 & 0.0005729 & 0.001913 & 0.008491 & $8.880 E-09$ & $3.532 E-08$ & $8.470 E-08$ & $1.094 E-06$ \\
4.5 & 0.000555 & 0.001558 & 0.000767 & 0.01375 & $2.018 E-07$ & $4.640 E-08$ & $3.321 E-07$ & $1.657 E-06$ \\
5.0 & 0.002561 & 0.004427 & $6.352 E-06$ & 0.02299 & $4.776 E-07$ & $7.358 E-07$ & $1.404 E-07$ & $3.066 E-06$ \\
\hline
\end{tabular}

$$
\begin{aligned}
& u_{1,2}(t) \\
& =\frac{1}{2}\left[a\left(-c_{1} c_{3}+c c_{2}\right)-a\left(a c_{2}-a c_{1}\right)\right] \\
& \times\left(t-t^{*}\right)^{2}, \\
& u_{2,2}(t) \\
& =\frac{1}{2}\left[-c_{1}\left(c_{1} c_{2}-b c_{3}\right)-c_{3}\left(a c_{2}-a c_{1}+c_{4}\right)\right. \\
& \left.+c\left(-c_{1} c_{3}+c c_{2}\right)\right]\left(t-t^{*}\right)^{2}, \\
& u_{2,3}(t) \\
& =\frac{1}{6}\left[-c_{1}\left(c_{1}\left(-c_{1} c_{3}+c c_{2}\right)+c_{2}\left(a c_{2}-a c_{1}+c_{4}\right)\right.\right. \\
& \left.-b\left(c_{1} c_{2}-b c_{3}\right)\right) \\
& -2\left(a c_{2}-a c_{1}+c_{4}\right)\left(c_{1} c_{2}-b c_{3}\right) \\
& -\left(a\left(-c_{1} c_{3}+c c_{2}\right)-a\left(a c_{2}-a c_{1}+c_{4}\right)\right. \\
& \left.+c_{1} c_{3}+r c_{4}\right) c_{4} \\
& +c\left(-c_{1}\left(c_{1} c_{2}-b c_{3}\right)-c_{3}\left(a c_{2}-a c_{1}+c_{4}\right)\right. \\
& \left.\left.+c\left(-c_{1} c_{3}+c c_{2}\right)\right)\right]\left(t-t^{*}\right)^{3}, \\
& u_{3,2}(t) \\
& =\frac{1}{2}\left[c_{1}\left(-c_{1} c_{3}+c c_{2}\right)+c_{2}\left(a c_{2}-a c_{1}+c_{4}\right)\right. \\
& \left.-b\left(c_{1} c_{2}-b c_{3}\right)\right]\left(t-t^{*}\right)^{2}, \\
& u_{3,3}(t) \\
& =\frac{1}{6}\left[c _ { 1 } \left(-c_{1}\left(c_{1} c_{2}-b c_{3}\right)-c_{3}\left(a c_{2}-a c_{1}+c_{4}\right)\right.\right. \\
& \left.+c\left(-c_{1} c_{3}+c c_{2}\right)\right) \\
& +2\left(a c_{2}-a c_{1}+c_{4}\right)\left(-c_{1} c_{3}+c c_{2}\right) \\
& +\left(a\left(-c_{1} c_{3}+c c_{2}\right)-a\left(a c_{2}-a c_{1}+c_{4}\right)\right. \\
& =\frac{1}{6}\left[a \left(-c_{1}\left(c_{1} c_{2}-b c_{3}\right)-c_{3}\left(a c_{2}-a c_{1}\right)\right.\right. \\
& \left.+c\left(-c_{1} c_{3}+c c_{2}\right)\right) \\
& -a\left(a\left(-c_{1} c_{3}+c c_{2}\right)\right. \\
& \left.\left.-a\left(a c_{2}-a c_{1}\right)\right)\right]\left(t-t^{*}\right)^{3}, \\
& \left.+c_{1} c_{3}+r c_{4}\right) c_{2} \\
& -b\left(c_{1}\left(-c_{1} c_{3}+c c_{2}\right)+c_{2}\left(a c_{2}-a c_{1}+c_{4}\right)\right. \\
& \left.\left.-b\left(c_{1} c_{2}-b c_{3}\right)\right)\right]\left(t-t^{*}\right)^{3} .
\end{aligned}
$$


TABLE 5: Differences between 15-term HPM and 15-term MHPM with RK4 solutions for $\Delta t=0.001$.

\begin{tabular}{|c|c|c|c|c|c|c|}
\hline \multirow[t]{2}{*}{$t$} & \multicolumn{3}{|c|}{$\Delta=\left|\mathrm{HPM}-\mathrm{RK}_{0.001}\right|$} & \multicolumn{3}{|c|}{$\Delta=\left|\mathrm{MHPM}_{0.001}-\mathrm{RK}_{0.001}\right|$} \\
\hline & $\Delta x$ & $\Delta y$ & $\Delta z$ & $\Delta x$ & $\Delta y$ & $\Delta z$ \\
\hline 0.5 & $4.986 E+08$ & $6.795 E+08$ & $7.897 E+08$ & $1.005 E-07$ & $7.882 E-08$ & $4.693 E-09$ \\
\hline 1.0 & $9.285 E+12$ & $1.284 E+13$ & $1.406 E+13$ & $3.371 E-08$ & $6.751 E-08$ & $1.256 E-07$ \\
\hline 1.5 & $2.838 E+15$ & $3.943 E+15$ & $4.219 E+15$ & $2.338 E-07$ & $2.362 E-07$ & $1.797 E-08$ \\
\hline 2.0 & $1.631 E+17$ & $2.271 E+17$ & $2.402 E+17$ & $1.034 E-07$ & $2.283 E-07$ & $8.887 E-07$ \\
\hline 2.5 & $3.763 E+18$ & $5.248 E+18$ & $5.506 E+18$ & $1.301 E-06$ & $1.048 E-06$ & $1.121 E-06$ \\
\hline 3.0 & $4.879 E+19$ & $6.810 E+19$ & $7.110 E+19$ & $1.941 E-06$ & $1.589 E-06$ & $3.214 E-07$ \\
\hline 3.5 & $4.252 E+20$ & $5.940 E+20$ & $6.178 E+20$ & $1.146 E-06$ & $1.377 E-06$ & $2.293 E-07$ \\
\hline 4.0 & $2.772 E+21$ & $3.874 E+21$ & $4.018 E+21$ & $1.564 E-05$ & $9.837 E-06$ & $1.147 E-05$ \\
\hline 4.5 & $1.448 E+22$ & $2.024 E+22$ & $2.095 E+22$ & $1.094 E-05$ & $1.735 E-05$ & $5.098 E-06$ \\
\hline 5.0 & $6.350 E+22$ & $8.882 E+22$ & $9.175 E+22$ & $5.727 E-05$ & $1.725 E-05$ & 0.0001507 \\
\hline
\end{tabular}

TABLE 6: Differences between 15-term HPM and 15-term MHPM with RK4 solutions on $\Delta t=0.001$.

\begin{tabular}{ccccccccc}
\hline$t$ & \multicolumn{4}{c}{$\Delta=\left|\mathrm{HPM}-\mathrm{RK} 4_{0.001}\right|$} & \multicolumn{3}{c}{$\Delta=\left|\mathrm{MHPM}_{0.001}-\mathrm{RK}_{0.001}\right|$} \\
& $\Delta x$ & $\Delta y$ & $\Delta z$ & $\Delta w$ & $\Delta x$ & $\Delta y$ & $\Delta z$ & $\Delta w$ \\
\hline 0.5 & $1.576 E+08$ & $1.073 E+10$ & $1.795 E+10$ & $1.108 E+10$ & $1.955 E-08$ & $1.067 E-08$ & $7.957 E-09$ & $6.043 E-08$ \\
1.0 & $7.248 E+12$ & $1.810 E+14$ & $3.311 E+14$ & $1.853 E+14$ & $2.300 E-09$ & $1.431 E-09$ & $9.090 E-09$ & $1.189 E-07$ \\
1.5 & $2.645 E+15$ & $5.321 E+16$ & $1.007 E+17$ & $5.428 E+16$ & $1.486 E-07$ & $1.683 E-07$ & $9.840 E-08$ & $1.346 E-07$ \\
2.0 & $1.642 E+17$ & $2.995 E+18$ & $5.767 E+18$ & $3.050 E+18$ & $4.049 E-08$ & $2.504 E-08$ & $1.678 E-07$ & $1.720 E-07$ \\
2.5 & $3.956 E+18$ & $6.821 E+19$ & $1.328 E+20$ & $6.939 E+19$ & $8.148 E-07$ & $1.303 E-06$ & $9.631 E-08$ & $1.632 E-06$ \\
3.0 & $5.274 E+19$ & $8.766 E+20$ & $1.719 E+21$ & $8.910 E+20$ & $3.607 E-07$ & $1.907 E-07$ & $3.559 E-07$ & $1.746 E-07$ \\
3.5 & $4.686 E+20$ & $7.592 E+21$ & $1.496 E+22$ & $7.713 E+21$ & $2.595 E-07$ & $1.474 E-07$ & $1.673 E-07$ & $3.415 E-07$ \\
4.0 & $3.099 E+21$ & $4.925 E+22$ & $9.747 E+22$ & $5.002 E+22$ & $5.266 E-08$ & $4.860 E-08$ & $1.174 E-07$ & $8.625 E-07$ \\
4.5 & $1.636 E+22$ & $2.563 E+23$ & $5.088 E+23$ & $2.602 E+23$ & $3.623 E-07$ & $4.214 E-07$ & $2.983 E-07$ & $1.357 E-06$ \\
5.0 & $7.239 E+22$ & $1.121 E+24$ & $2.230 E+24$ & $1.137 E+24$ & $1.576 E-06$ & $1.956 E-06$ & $6.830 E-07$ & $2.405 E-07$ \\
\hline
\end{tabular}

Thus, the solution of system (1) is

$$
\begin{aligned}
& x(t)=\sum_{k=0}^{\infty} u_{1, k}(t), \\
& y(t)=\sum_{k=0}^{\infty} u_{2, k}(t), \\
& z(t)=\sum_{k=0}^{\infty} u_{3, k}(t) .
\end{aligned}
$$

To carry out the iterations on every subinterval of equal length $\Delta t$, we need to know the values of the following initial conditions:

$$
c_{1}=x\left(t^{*}\right), \quad c_{2}=y\left(t^{*}\right), \quad c_{3}=z\left(t^{*}\right) .
$$

In general, we do not have this information except at the initial point $t^{*}=t_{0}=0$, but we can obtain these values following the MHPM as discussed earlier. We note that the 15-term approximations of $x, y, z$, and $w$ are denoted as

$$
\begin{aligned}
& x(t) \approx \phi_{15}(t)=\sum_{i=0}^{14} u_{1, i}, \\
& y(t) \approx \phi_{15}(t)=\sum_{i=0}^{14} u_{2, i}, \\
& z(t) \approx \phi_{15}(t)=\sum_{i=0}^{14} u_{3, i} .
\end{aligned}
$$

3.2. Hyperchaotic Lü System. The hyperchaotic Lü system (2) is subject to initial conditions

$$
\begin{array}{ll}
x\left(t_{0}\right)=c_{1}, & y\left(t_{0}\right)=c_{2}, \\
z\left(t_{0}\right)=c_{3}, & w\left(t_{0}\right)=c_{4} .
\end{array}
$$


TABLE 7: Differences between 15-term HPM and 15-term MHPM with RK4 solutions on $\Delta t=0.001$.

\begin{tabular}{|c|c|c|c|c|c|c|c|c|}
\hline \multirow{2}{*}{$t$} & \multicolumn{4}{|c|}{$\Delta=\left|\mathrm{HPM}-\mathrm{RK} 4_{0.001}\right|$} & \multicolumn{4}{|c|}{$\Delta=\left|\mathrm{MHPM}_{0.001}-\mathrm{RK}_{0.001}\right|$} \\
\hline & $\Delta x$ & $\Delta y$ & $\Delta z$ & $\Delta w$ & $\Delta x$ & $\Delta y$ & $\Delta z$ & $\Delta w$ \\
\hline 0.5 & $1.623 E+09$ & $4.852 E+09$ & $4.416 E+09$ & $3.649 E+09$ & $2.402 E-07$ & $1.477 E-07$ & $1.948 E-07$ & $4.362 E-07$ \\
\hline 1.0 & $2.235 E+13$ & $6.330 E+13$ & $7.013 E+13$ & $4.647 E+13$ & $3.230 E-07$ & $1.671 E-07$ & $5.436 E-07$ & $1.878 E-06$ \\
\hline 1.5 & $6.166 E+15$ & $1.717 E+16$ & $2.013 E+16$ & $1.251 E+16$ & $6.006 E-07$ & $2.847 E-07$ & $9.289 E-07$ & $1.075 E-06$ \\
\hline 2.0 & $3.365 E+17$ & $9.294 E+17$ & $1.119 E+18$ & $6.744 E+17$ & $1.539 E-06$ & $3.304 E-07$ & $4.160 E-06$ & $3.999 E-06$ \\
\hline 2.5 & $7.525 E+18$ & $2.068 E+19$ & $2.531 E+19$ & $1.497 E+19$ & $1.137 E-06$ & $1.669 E-06$ & $3.667 E-06$ & $9.757 E-06$ \\
\hline 3.0 & $9.556 E+19$ & $2.618 E+20$ & $3.236 E+20$ & $1.892 E+20$ & $2.159 E-06$ & $2.944 E-06$ & $2.533 E-06$ & $1.780 E-05$ \\
\hline 3.5 & $8.206 E+20$ & $2.243 E+21$ & $2.793 E+21$ & $1.619 E+21$ & $1.230 E-05$ & $1.589 E-05$ & $4.910 E-06$ & $1.254 E-05$ \\
\hline 4.0 & $5.290 E+21$ & $1.443 E+22$ & $1.807 E+22$ & $1.041 E+22$ & $3.463 E-05$ & $2.798 E-05$ & $7.285 E-05$ & 0.0002694 \\
\hline 4.5 & $2.739 E+22$ & $7.464 E+22$ & $9.385 E+22$ & $5.381 E+22$ & $3.844 E-05$ & $3.378 E-05$ & $3.462 E-05$ & 0.0001885 \\
\hline 5.0 & $1.193 E+23$ & $3.247 E+23$ & $4.097 E+23$ & $2.340 E+23$ & $3.208 E-05$ & $4.220 E-05$ & $2.855 E-07$ & 0.0002054 \\
\hline
\end{tabular}

TABLE 8: Differences between 15-term HPM and 15-term MHPM with RK4 solutions on $\Delta t=0.001$.

\begin{tabular}{|c|c|c|c|c|c|c|c|c|}
\hline \multirow{2}{*}{$t$} & \multicolumn{4}{|c|}{$\Delta=\left|\mathrm{HPM}-\mathrm{RK} 4_{0.001}\right|$} & \multicolumn{4}{|c|}{$\Delta=\left|\mathrm{MHPM}_{0.001}-\mathrm{RK}_{0.001}\right|$} \\
\hline & $\Delta x$ & $\Delta y$ & $\Delta z$ & $\Delta w$ & $\Delta x$ & $\Delta y$ & $\Delta z$ & $\Delta w$ \\
\hline 0.5 & $1.804 E+09$ & $8.725 E+09$ & $2.906 E+10$ & $8.597 E+09$ & $2.629 E-07$ & $6.162 E-07$ & $2.124 E-07$ & $1.026 E-06$ \\
\hline 1.0 & $3.807 E+13$ & $1.427 E+14$ & $5.243 E+14$ & $1.383 E+14$ & $4.476 E-07$ & $4.547 E-07$ & $2.484 E-07$ & $6.317 E-07$ \\
\hline 1.5 & $1.213 E+16$ & $4.143 E+16$ & $1.583 E+17$ & $3.987 E+16$ & $2.166 E-07$ & $4.588 E-07$ & $2.687 E-07$ & $9.034 E-07$ \\
\hline 2.0 & $7.114 E+17$ & $2.316 E+18$ & $9.040 E+18$ & $2.220 E+18$ & $3.632 E-07$ & $3.035 E-07$ & $7.236 E-07$ & $8.202 E-07$ \\
\hline 2.5 & $1.661 E+19$ & $5.252 E+19$ & $2.077 E+20$ & $5.022 E+19$ & $3.281 E-07$ & $3.616 E-07$ & $1.068 E-07$ & $7.918 E-07$ \\
\hline 3.0 & $2.171 E+20$ & $6.731 E+20$ & $2.685 E+21$ & $6.424 E+20$ & $3.620 E-07$ & $1.322 E-07$ & $1.181 E-06$ & $9.254 E-07$ \\
\hline 3.5 & $1.904 E+21$ & $5.816 E+21$ & $2.336 E+22$ & $5.544 E+21$ & $1.053 E-07$ & $1.528 E-07$ & $4.943 E-08$ & $8.159 E-07$ \\
\hline 4.0 & $1.246 E+22$ & $3.767 E+22$ & $1.521 E+23$ & $3.587 E+22$ & $8.876 E-09$ & $3.532 E-08$ & $8.471 E-08$ & $1.094 E-06$ \\
\hline 4.5 & $6.532 E+22$ & $1.958 E+23$ & $7.933 E+23$ & $1.863 E+23$ & $2.018 E-07$ & $4.640 E-08$ & $3.321 E-07$ & $1.657 E-06$ \\
\hline 5.0 & $2.872 E+23$ & $8.551 E+23$ & $3.476 E+24$ & $8.131 E+23$ & $4.776 E-07$ & $7.359 E-07$ & $1.404 E-07$ & $3.066 E-06$ \\
\hline
\end{tabular}

According to HPM, we can construct a homotopy which satisfies the following relations:

$$
\begin{gathered}
\dot{u}_{1}-\dot{x}_{0}+p\left(\dot{x}_{0}-a u_{2}+a u_{1}-u_{4}\right)=0 \\
\dot{u}_{2}-\dot{y}_{0}+p\left(\dot{y}_{0}+u_{1} u_{3}-c u_{2}\right)=0 \\
\dot{u}_{3}-\dot{z}_{0}+p\left(\dot{z}_{0}-u_{1} u_{2}+b u_{3}\right)=0 \\
\dot{u}_{4}-\dot{w}_{0}+p\left(\dot{w}_{0}-u_{1} u_{3}-r u_{4}\right)=0
\end{gathered}
$$

We take the initial approximations as

$$
\begin{gathered}
u_{1,0}(t)=x_{0}(t)=x\left(t^{*}\right)=c_{1}, \\
u_{2,0}(t)=y_{0}(t)=y\left(t^{*}\right)=c_{2}, \\
u_{3,0}(t)=z_{0}(t)=z\left(t^{*}\right)=c_{3}, \\
u_{4,0}(t)=w_{0}(t)=w\left(t^{*}\right)=c_{4}, \\
u_{1}(t)=u_{1,0}(t)+p u_{1,1}(t)+p^{2} u_{1,2}(t)+p^{3} u_{1,3}(t)+\cdots, \\
u_{2}(t)=u_{2,0}(t)+p u_{2,1}(t)+p^{2} u_{2,2}(t)+p^{3} u_{2,3}(t)+\cdots, \\
u_{3}(t)=u_{3,0}(t)+p u_{3,1}(t)+p^{2} u_{3,2}(t)+p^{3} u_{3,3}(t)+\cdots, \\
u_{4}(t)=u_{4,0}(t)+p u_{4,1}(t)+p^{2} u_{4,2}(t)+p^{3} u_{4,3}(t)+\cdots,
\end{gathered}
$$

where $u_{i, j}(t), i=j=1,2,3, \ldots$ are functions yet to be determined. Substituting (25) into (24) and arranging the terms with the same powers of $p$, we have

$$
\begin{gathered}
\dot{u}_{1,1}-a u_{2,0}+a u_{1,0}-u_{4,0}=0, \\
\dot{u}_{1,2}-a u_{2,1}+a u_{1,1}-u_{4,1}=0, \\
\dot{u}_{1,3}-a u_{2,2}+a u_{1,2}-u_{4,2}=0 \\
\dot{u}_{2,1}+u_{1,0} u_{3,0}-c u_{2,0}=0, \\
\dot{u}_{2,2}+u_{1,0} u_{3,1}+u_{1,1} u_{3,0}-c u_{2,1}=0, \\
\dot{u}_{2,3}+u_{1,0} u_{3,2}+u_{1,1} u_{3,1}+u_{1,2} u_{3,0}-c u_{2,2}=0, \\
\dot{u}_{3,1}-u_{1,0} u_{2,0}+b u_{3,0}=0, \\
\dot{u}_{3,2}-u_{1,0} u_{2,1}-u_{1,1} u_{2,0}+b u_{3,1}=0, \\
\dot{u}_{3,3}-u_{1,0} u_{2,2}-u_{1,1} u_{2,1}-u_{1,2} u_{2,0}+b u_{3,2}=0, \\
\dot{u}_{4,1}-u_{1,0} u_{3,0}-r u_{4,0}=0 \\
\dot{u}_{4,2}-u_{1,0} u_{3,1}-u_{1,1} u_{3,0}-r u_{4,1}=0, \\
\dot{u}_{4,3}-u_{1,0} u_{3,2}-u_{1,1} u_{3,1}-u_{1,2} u_{3,0}-r u_{4,2}=0 .
\end{gathered}
$$




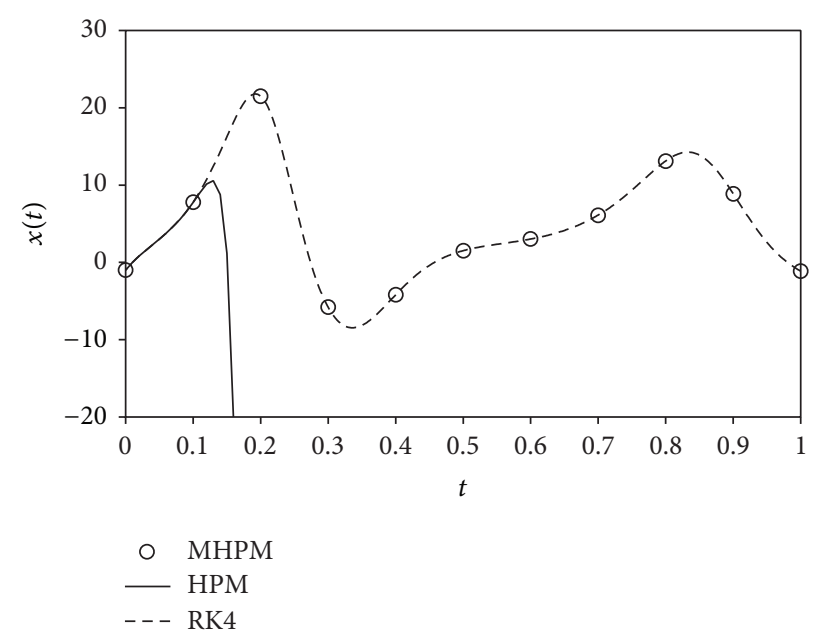

(a)

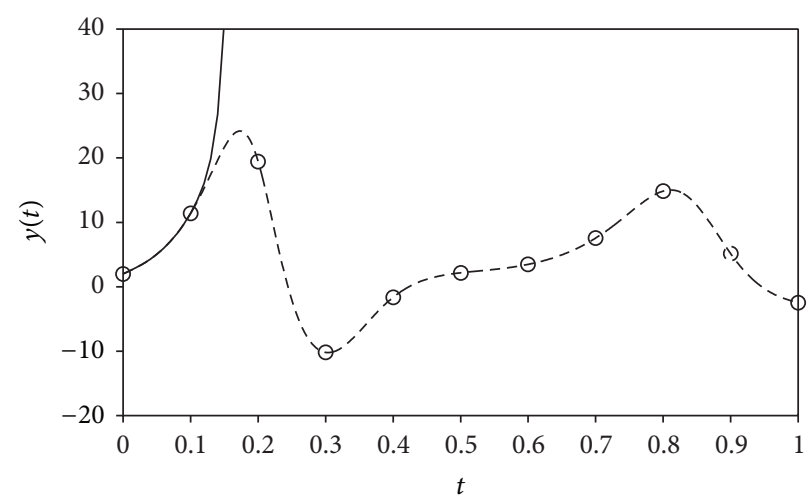

○ MHPM

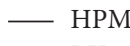

-.- RK4

(b)

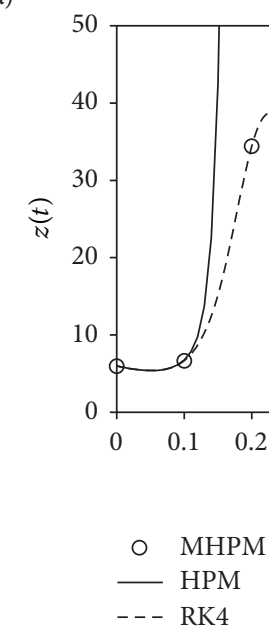

(c)

FIGURE 1: Comparison among 15-term solutions of HPM (solid line), MHPM (circle), and RK4 (dash line) for chaotic Lü system.

Solve for the unknowns of (26) by taking the initial conditions

as $u_{i, j}(0)=0, i, j=1,2,3$

$$
\begin{array}{cc}
u_{1,1}(t)=\left[a c_{2}-a c_{1}+c_{4}\right]\left(t-t^{*}\right), & =\frac{1}{2}\left[c_{1}\left(-c_{1} c_{3}+c c_{2}\right)+c_{2}\left(a c_{2}-a c_{1}+c_{4}\right)\right. \\
u_{2,1}(t)=\left[-c_{1} c_{3}+c c_{2}\right]\left(t-t^{*}\right), & \left.-b\left(c_{1} c_{2}-b c_{3}\right)\right]\left(t-t^{*}\right)^{2}, \\
u_{3,1}(t)=\left[c_{1} c_{2}-b c_{3}\right]\left(t-t^{*}\right), & u_{4,2}(t) \\
u_{4,1}(t)=\left[c_{1} c_{3}+r c_{4}\right]\left(t-t^{*}\right), & =\frac{1}{2}\left[c_{1}\left(c_{1} c_{2}-b c_{3}\right)+c_{3}\left(a c_{2}-a c_{1}+c_{4}\right)\right. \\
u_{1,2}(t) & \left.+r\left(c_{1} c_{3}-r c_{4}\right)\right]\left(t-t^{*}\right)^{2}, \\
=\frac{1}{2}\left[a\left(-c_{1} c_{3}+c c_{2}\right)-a\left(a c_{2}-a c_{1}+c_{4}\right)\right. & \\
\left.+c_{1} c_{3}+r c_{4}\right]\left(t-t^{*}\right)^{2}, & u_{1,3}(t) \\
u_{2,2}(t) \quad & =\frac{1}{6}\left[a \left(-c_{1}\left(c_{1} c_{2}-b c_{3}\right)-c_{3}\left(a c_{2}-a c_{1}+c_{4}\right)\right.\right. \\
=\frac{1}{2}\left[-c_{1}\left(c_{1} c_{2}-b c_{3}\right)-c_{3}\left(a c_{2}-a c_{1}+c_{4}\right)\right. & \left.+c\left(-c_{1} c_{3}+c c_{2}\right)\right)
\end{array}
$$




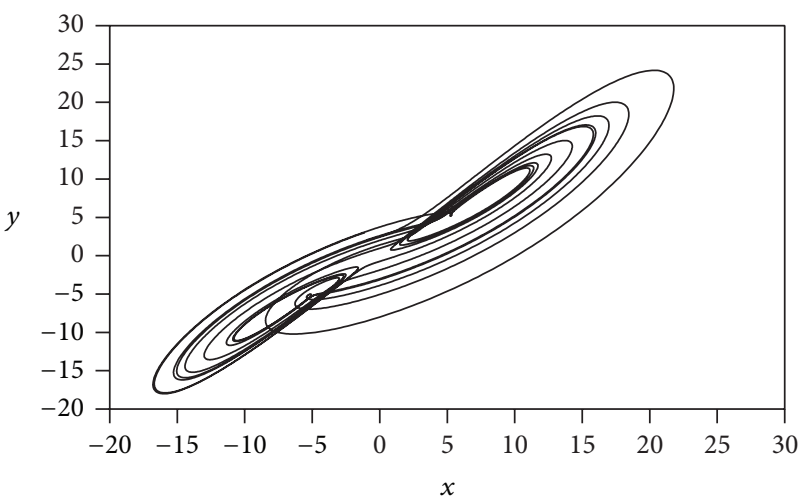

(a)

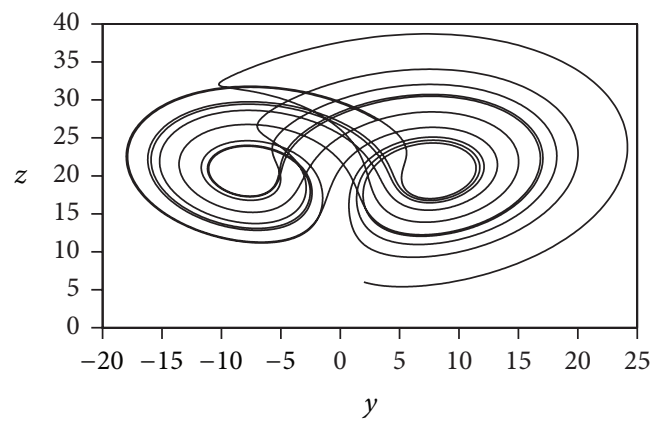

(c)

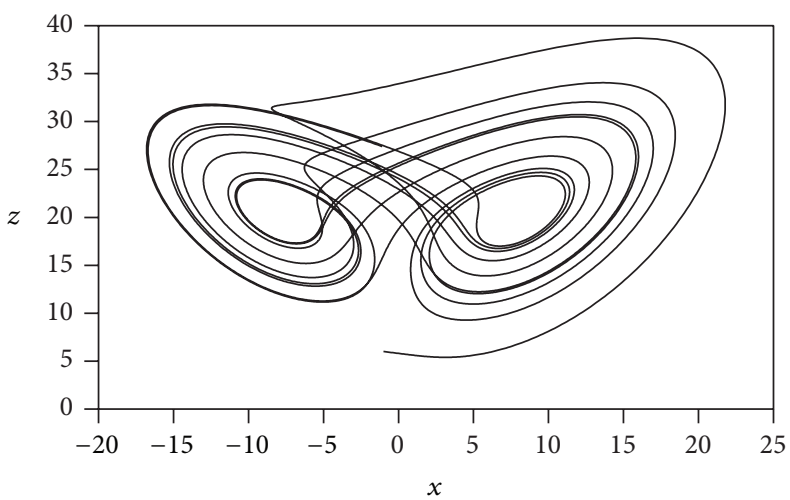

(b)

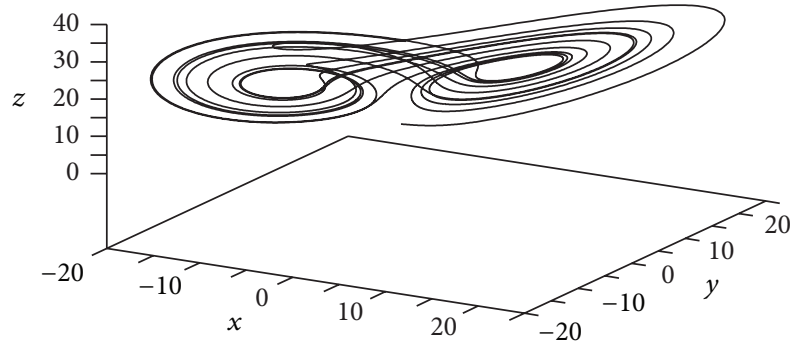

(d)

Figure 2: Phase portraits of 15-term MHPM of Chaotic Lü system.

$$
\begin{aligned}
& -a\left(a\left(-c_{1} c_{3}+c c_{2}\right)-a\left(a c_{2}-a c_{1}+c_{4}\right)\right. \\
& \left.\quad+c_{1} c_{3}+r c_{4}\right) \\
& +c_{1}\left(c_{1} c_{2}-b c_{3}\right)+c_{3}\left(a c_{2}-a c_{1}+c_{4}\right) \\
& \left.+r\left(c_{1} c_{3}-r c_{4}\right)\right]\left(t-t^{*}\right)^{3},
\end{aligned}
$$

$u_{2,3}(t)$

$$
=\frac{1}{6}\left[-c_{1}\left(c_{1}\left(-c_{1} c_{3}+c c_{2}\right)+c_{2}\left(a c_{2}-a c_{1}+c_{4}\right)\right.\right.
$$$$
\left.-b\left(c_{1} c_{2}-b c_{3}\right)\right)
$$$$
-2\left(a c_{2}-a c_{1}+c_{4}\right)\left(c_{1} c_{2}-b c_{3}\right)
$$$$
-\left(a\left(-c_{1} c_{3}+c c_{2}\right)-a\left(a c_{2}-a c_{1}+c_{4}\right)\right.
$$$$
\left.+c_{1} c_{3}+r c_{4}\right) c_{4}
$$$$
+c\left(-c_{1}\left(c_{1} c_{2}-b c_{3}\right)-c_{3}\left(a c_{2}-a c_{1}+c_{4}\right)\right.
$$$$
\left.\left.+c\left(-c_{1} c_{3}+c c_{2}\right)\right)\right]\left(t-t^{*}\right)^{3},
$$

$$
\begin{aligned}
& u_{3,3}(t) \\
& =\frac{1}{6}\left[c _ { 1 } \left(-c_{1}\left(c_{1} c_{2}-b c_{3}\right)-c_{3}\left(a c_{2}-a c_{1}+c_{4}\right)\right.\right. \\
& \left.+c\left(-c_{1} c_{3}+c c_{2}\right)\right) \\
& +2\left(a c_{2}-a c_{1}+c_{4}\right)\left(-c_{1} c_{3}+c c_{2}\right) \\
& +\left(a\left(-c_{1} c_{3}+c c_{2}\right)-a\left(a c_{2}-a c_{1}+c_{4}\right)\right. \\
& \left.+c_{1} c_{3}+r c_{4}\right) c_{2} \\
& -b\left(c_{1}\left(-c_{1} c_{3}+c c_{2}\right)+c_{2}\left(a c_{2}-a c_{1}+c_{4}\right)\right. \\
& \left.\left.-b\left(c_{1} c_{2}-b c_{3}\right)\right)\right]\left(t-t^{*}\right)^{3}, \\
& u_{4,3}(t) \quad \begin{array}{c}
1 \\
6
\end{array} \\
& c_{1}\left(c_{1}\left(-c_{1} c_{3}+c c_{2}\right)+c_{2}\left(a c_{2}-a c_{1}+c_{4}\right)\right. \\
& \left.-b\left(c_{1} c_{2}-b c_{3}\right)\right) \\
& +2\left(a c_{2}-a c_{1}+c_{4}\right)\left(c_{1} c_{2}-b c_{3}\right) \\
& +\left(a\left(-c_{1} c_{3}+c c_{2}\right)-a\left(a c_{2}-a c_{1}+c_{4}\right)\right. \\
& \left.+c_{1} c_{3}+r c_{4}\right) c_{4}
\end{aligned}
$$




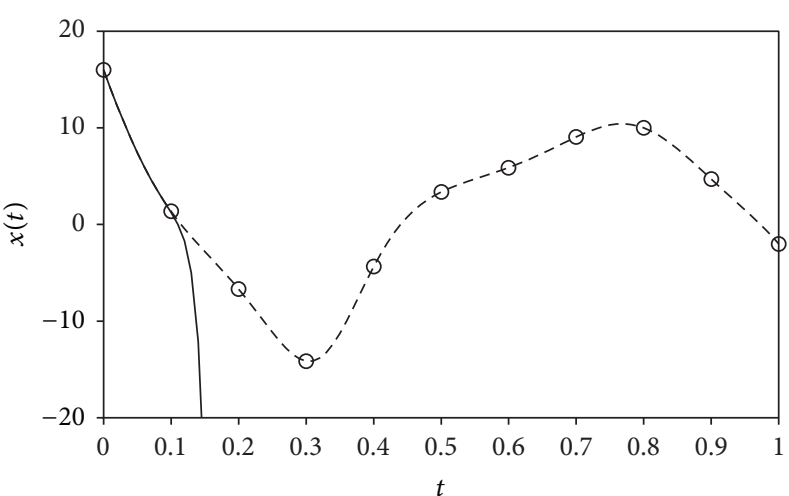

(a)
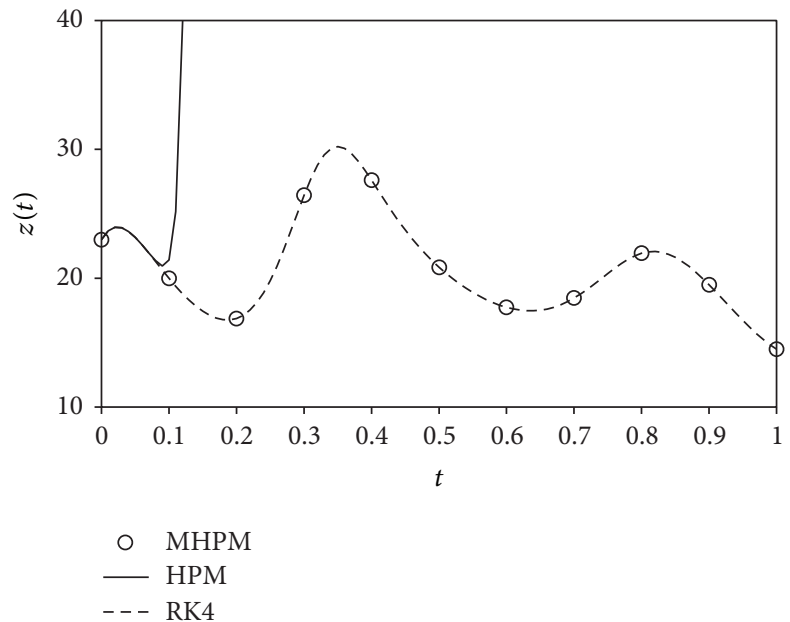

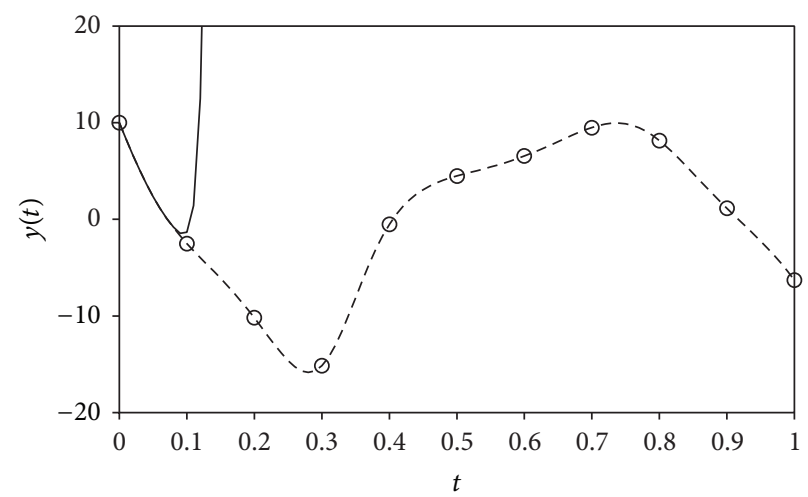

(b)
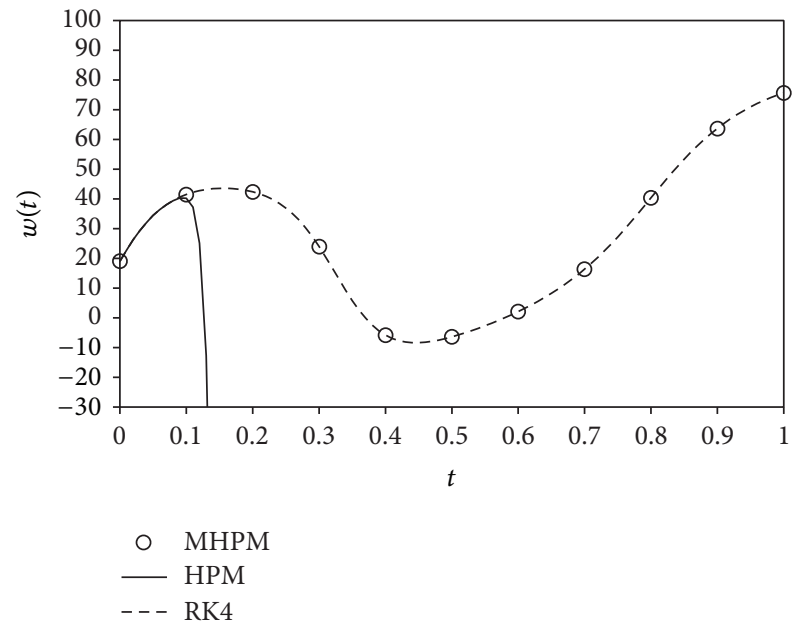

(c)

(d)

FIGURE 3: Comparison among 15-term HPM (solid line), MHPM (circle), and RK4 (dash line) for Case 1 of hyperchaotic Lü system.

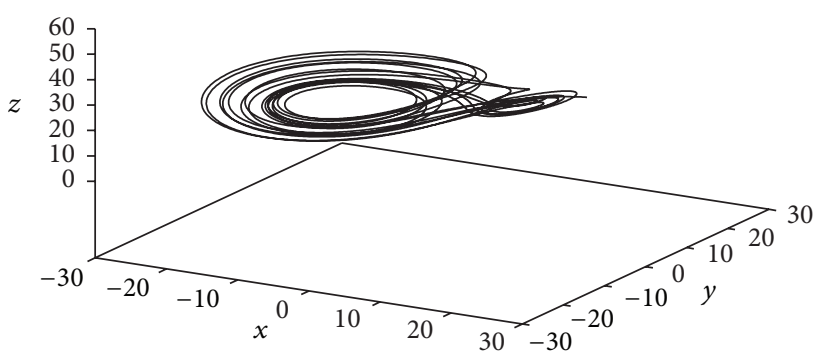

(a)

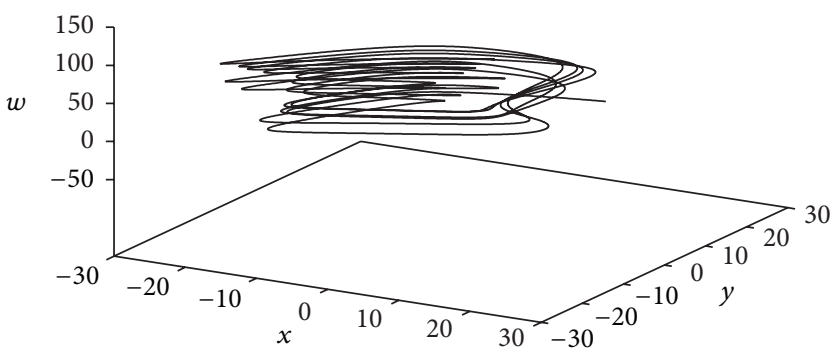

(c)

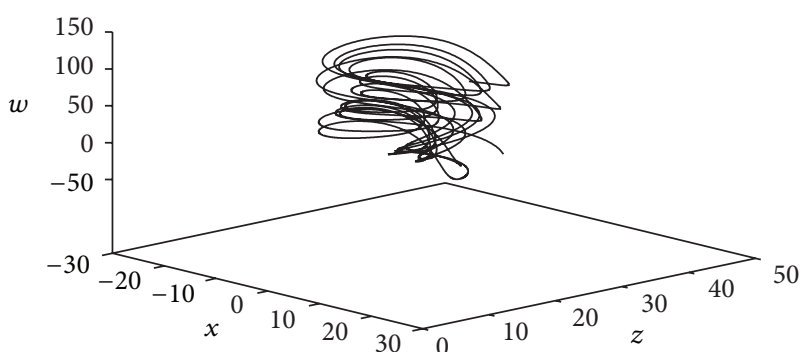

(b)

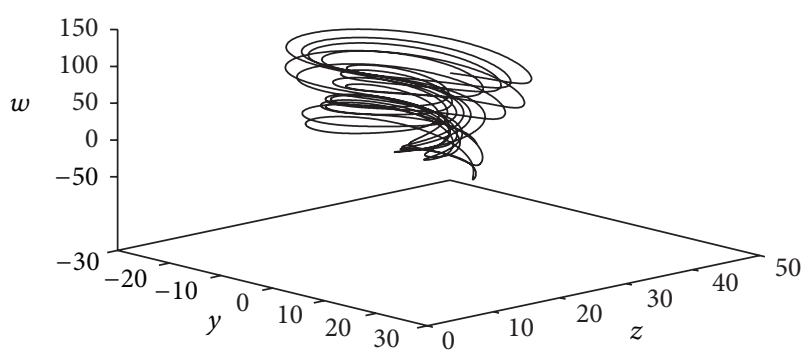

(d)

FIgURE 4: Phase portraits using 15-term MHPM on $\Delta t=0.001$ of Case 1 hyperchaotic Lü system. 


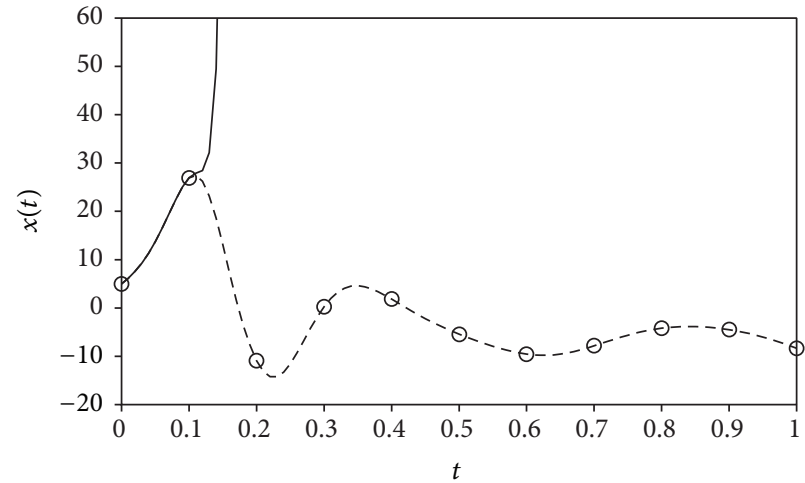

(a)

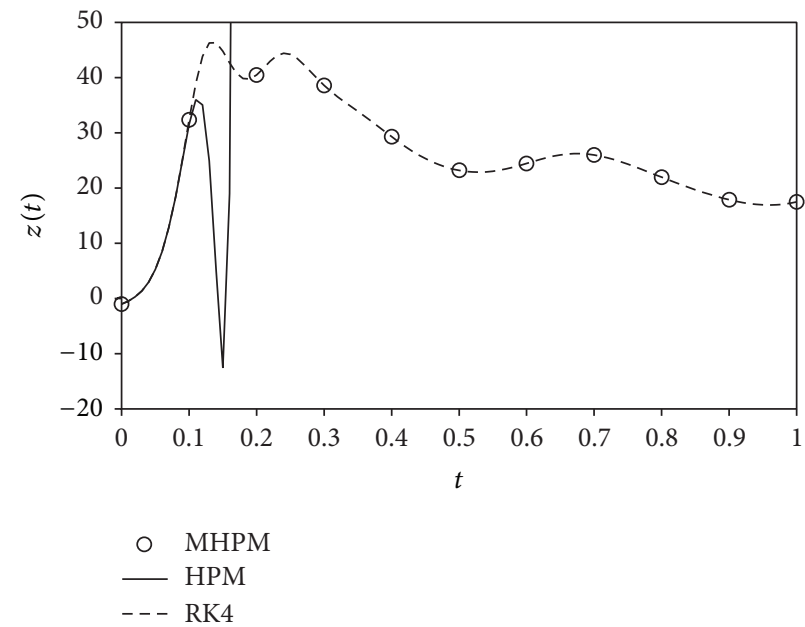

(c)

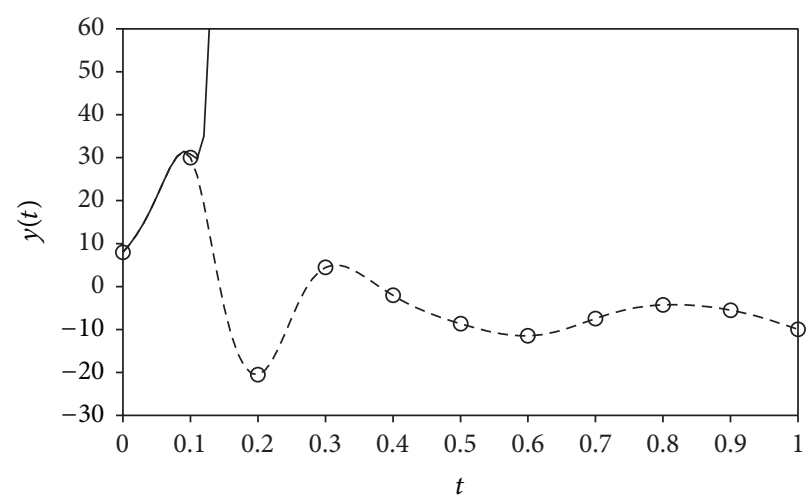

(b)

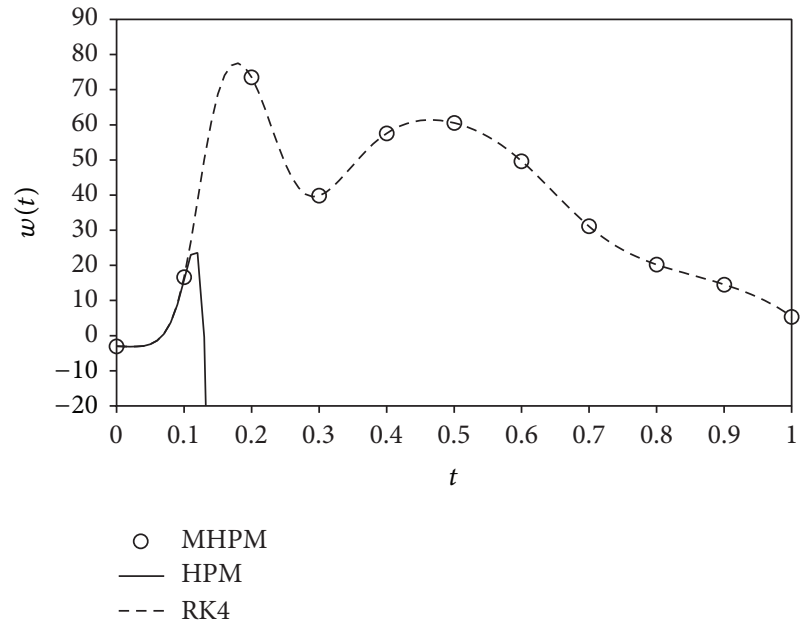

(d)

FIgURE 5: Comparison among 15-term HPM (solid line), MHPM (circle), and RK4 (dash line) for Case (2) of hyperchaotic Lü system.

$$
\begin{aligned}
& +r\left(c_{1}\left(c_{1} c_{2}-b c_{3}\right)+c_{3}\left(a c_{2}-a c_{1}+c_{4}\right)\right. \\
& \left.\left.\quad+r\left(c_{1} c_{3}-r c_{4}\right)\right)\right]\left(t-t^{*}\right)^{3} .
\end{aligned}
$$

Thus, the solution of system (2) is

$$
\begin{aligned}
& x(t)=\sum_{k=0}^{\infty} u_{1, k}(t), \\
& y(t)=\sum_{k=0}^{\infty} u_{2, k}(t), \\
& z(t)=\sum_{k=0}^{\infty} u_{3, k}(t), \\
& w(t)=\sum_{k=0}^{\infty} u_{4, k}(t) .
\end{aligned}
$$

To carry out the iterations on every subinterval of equal length $\Delta t$, we need to know the values of the following initial conditions:

$$
\begin{array}{ll}
c_{1}=x\left(t^{*}\right), & c_{2}=y\left(t^{*}\right), \\
c_{3}=z\left(t^{*}\right), & c_{4}=w\left(t^{*}\right) .
\end{array}
$$

In general, we do not have this information except at the initial point $t^{*}=t_{0}=0$, but we can obtain these values following the MHPM as discussed earlier. We note that the 15-term approximations of $x, y, z$, and $w$ are denoted as

$$
\begin{aligned}
& x(t) \approx \phi_{15}(t)=\sum_{i=0}^{14} u_{1, i}, \\
& y(t) \approx \phi_{15}(t)=\sum_{i=0}^{14} u_{2, i},
\end{aligned}
$$




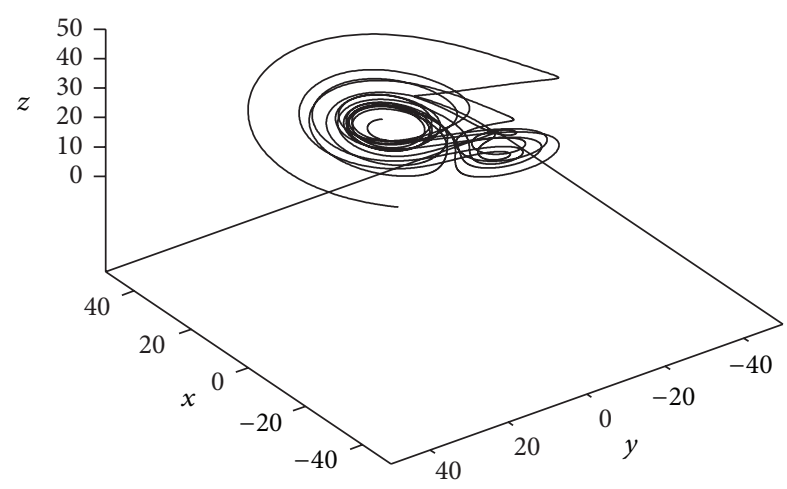

(a)

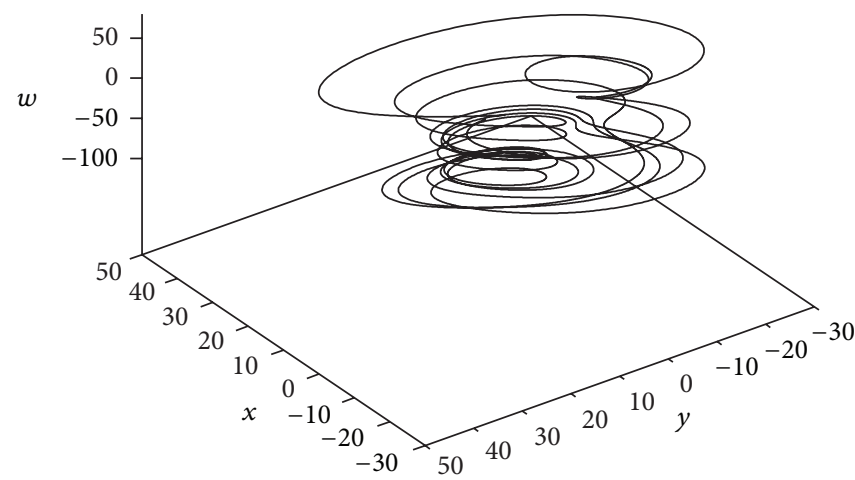

(c)

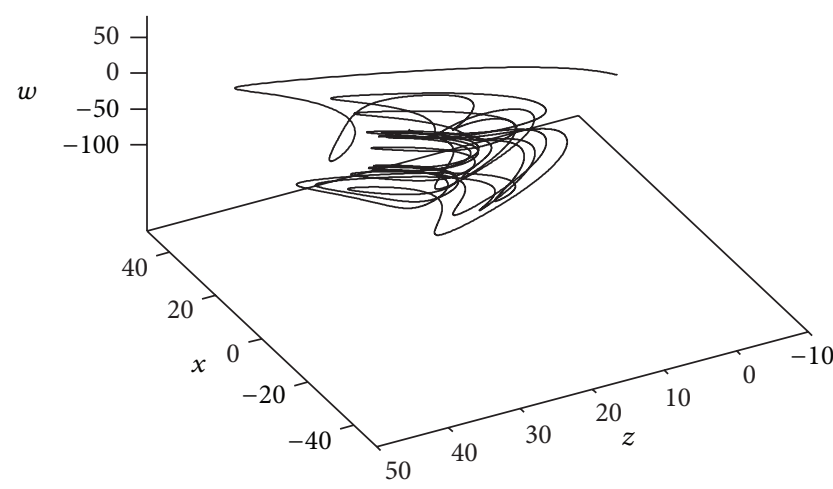

(b)

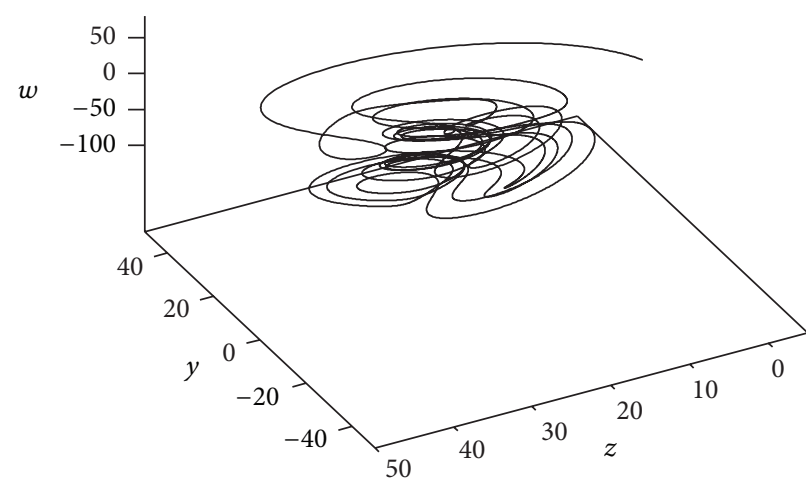

(d)

Figure 6: Phase portraits using 15-term MHPM on $\Delta t=0.001$ of Case 2 hyperchaotic Lü system.

$$
\begin{aligned}
& z(t) \approx \phi_{15}(t)=\sum_{i=0}^{14} u_{3, i}, \\
& w(t) \approx \phi_{15}(t)=\sum_{i=0}^{14} u_{4, i} .
\end{aligned}
$$

\section{Results and Discussions}

The MHPM algorithm is coded in the computer algebra package Maple together with the Maple built-in fourthorder Runge-Kutta. The Maple environment variable digits controller is set to 16 in all calculations. Time range is from 0 to 5 and the number of terms used is fixed to 15 . As an example for the hyperchaotic Lü system, we solve it for three different sets of initial conditions which are Case (1); $\left(x_{0}, y_{0}, z_{0}, w_{0}\right)=$ $(16,10,19,23)$, Case $(2) ;\left(x_{0}, y_{0}, z_{0}, w_{0}\right)=(5,8,-1,-3)$, and Case (3); $\left(x_{0}, y_{0}, z_{0}, w_{0}\right)=(6,0.5,4,-9)$. For the selection of time step to be used for the entire work, we determine it by comparing the solution of fourth-order Runge-Kutta (RK4) for the time increments $\Delta t=0.01, \Delta t=0.001$, and $\Delta t=$ 0.0001 . From the data presented in Table 1 for the chaotic Lü system and Tables 2, 3, and 4 for hyperchaotic Lü system, we see that the differences between $\Delta t=0.001$ and $\Delta t=0.0001$ are smaller than the differences between $\Delta t=0.01$ and $\Delta t=$ 0.001 . This shows that $\Delta t=0.001$ and $\Delta t=0.0001$ give better accuracy than $\Delta t=0.01$. Based on this observation, we choose $\Delta t=0.001$ as it consumes less time. Since there are no exact solutions for the Lü system, the results of HPM and MHPM are compared to the existing numerical solutions obtained by applying the fourth-order Runge-Kutta scheme. Tables 5, 6, 7, and 8 show the comparison of HPM and MHPM with RK4.

4.1. Chaotic Lü System. For the chaotic Lü system, the parameters involved are $a=36, b=3$, and $c=20$ alongside the initial conditions of $x_{0}=-1, y=2$, and $z=6$. The 15term HPM solutions for the chaotic Lü system are obtained as follows:

$$
\begin{aligned}
x= & -1.0+108.0 t-1116.000000 t^{2} \\
& +14904.00000 t^{3}-99663.00000 t^{4} \\
& +633329.4000 t^{5}-2953565.400 t^{6} \\
& +12353885.16 t^{7}-57191750.94 t^{8} \\
& +348616602.2 t^{9}-3132645470 t^{10} \\
& +27260231260 t^{11}-224688814200 t^{12} \\
& +1637561100000 t^{13}-10722147350000 t^{14},
\end{aligned}
$$




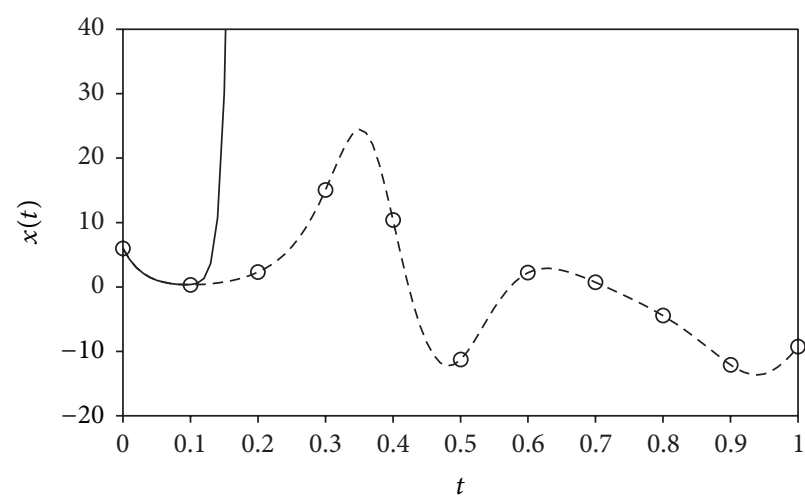

(a)

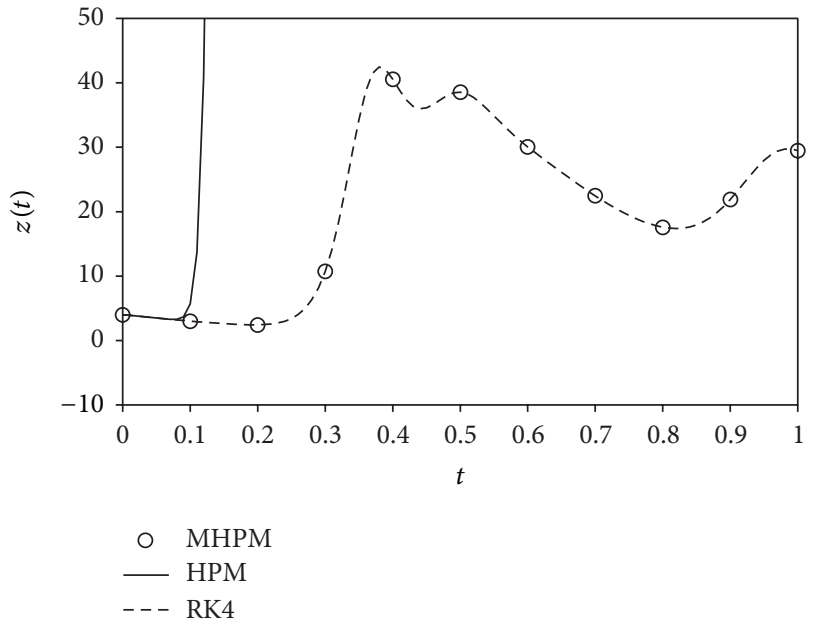

(c)

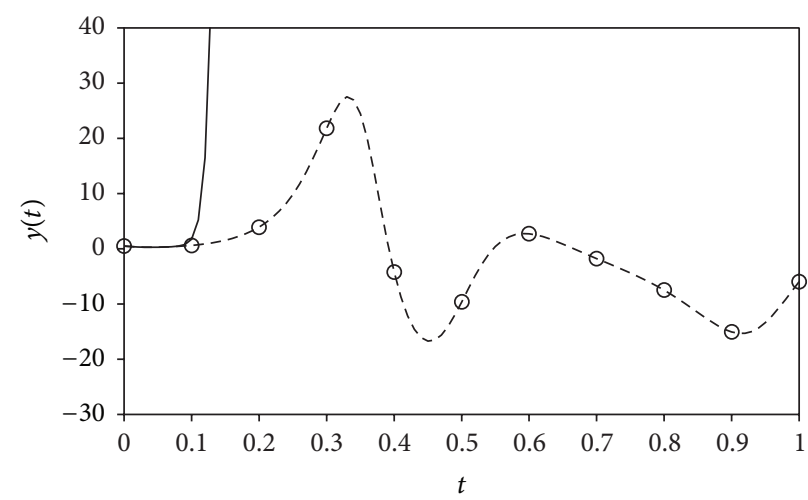

(b)

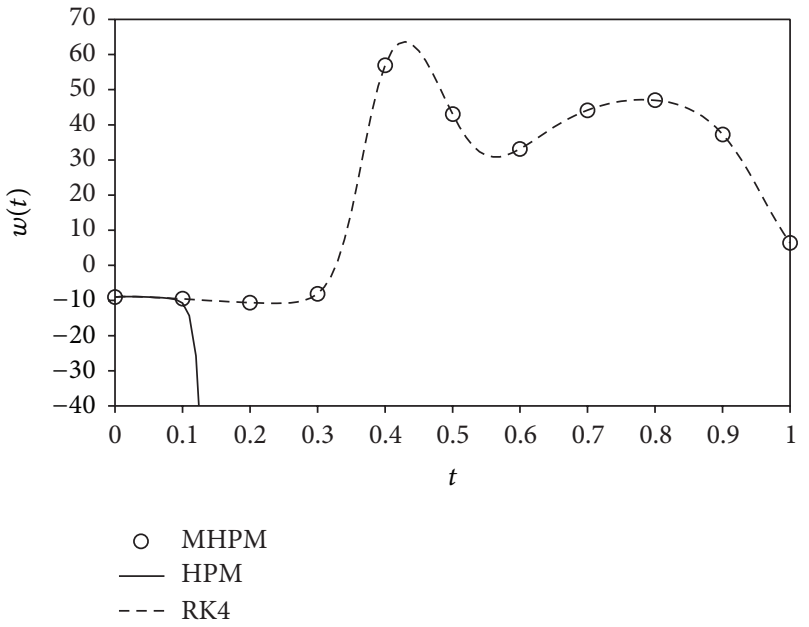

(d)

FIgURE 7: Comparison among 15-term HPM (solid line), MHPM (circle), and RK4 (dash line) for Case (3) of hyperchaotic Lü system.

$$
\begin{aligned}
y= & 2.0+46.00 t+126.0000000 t^{2} \\
& +3830.333333 t^{3}-11700.58333 t^{4} \\
& +141068.5000 t^{5}-551421.0639 t^{6} \\
& -355392.8282 t^{7}+29962399.58 t^{8} \\
& -521562694.8 t^{9}+5196869637 t^{10} \\
& -47636040140 t^{11}+366652694300 t^{12} \\
& -2532162870000 t^{13}+15044012470000 t^{14}, \\
z= & 6.0-20.00 t+115.0000000 t^{2} \\
& +755.000000 t^{3}-3503.833332 t^{4} \\
& +156306.0167 t^{5}-1264706.125 t^{6} \\
& +14342080.01 t^{7}-105862688.4 t^{8} \\
& +737880888.2 t^{9}-3943767312 t^{10} \\
& +12755709360 t^{11}+38293419600 t^{12} \\
& -1278826847000 t^{13}+15286095180000 t^{14} .
\end{aligned}
$$

In Table 5, we compare the solutions of HPM and MHPM to the RK4. Based on the negligible differnces between the two solutions we observe that MHPM solves the chaotic Lü system accurately. As for HPM, it diverges rapidly thus far from the RK4 solution even at $t=0.5$. To see clearly at what point HPM begins to diverge, we plot all the solutions of HPM, MHPM, and RK4 into a graph as shown in Figure 1, where HPM starts to diverge at $t<0.2 \mathrm{~s}$. Then, we reproduce the $x-y, x-z, y-z$, and $x-y-z$ phase portraits of 15 -term MHPM for chaotic Lü system in Figure 2 which shows their smooth behaviour in the phase plane.

4.2. Hyperchaotic Lü System. In this section, we solve the hyperchaotic Lü system with the parameters of $a=36, b=3$, $c=20$, and $r=1.3$. We discuss three sets of different initial conditions to prove that MHPM is helpful in solving the hyperchaotic Lü system accurately.

4.2.1. Case 1. Subject to initial conditions, $\left(x_{0}, y_{0}, z_{0}, w_{0}\right)=$ $(16,10,19,23)$, (Figures 3 and 4$)$ the 15-term solutionc obtained by applying the HPM on the chaotic Lü system are

$$
x(t)=16.0-197.0 t+718.3500000 t^{2}
$$

$$
-10757.61500 t^{3}+207284.0626 t^{4}
$$




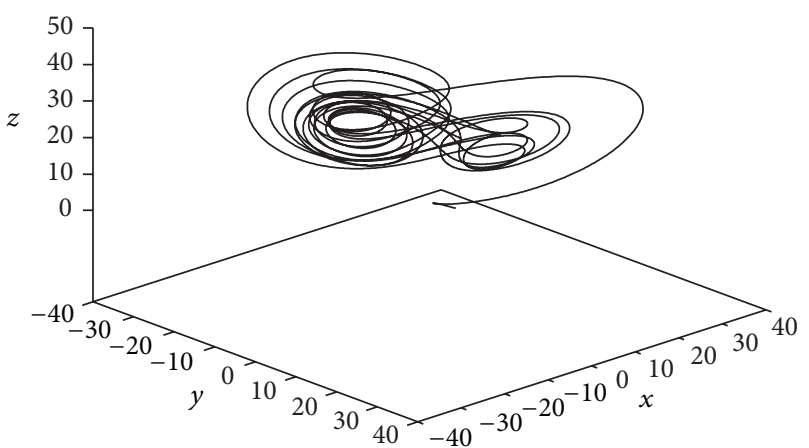

(a)

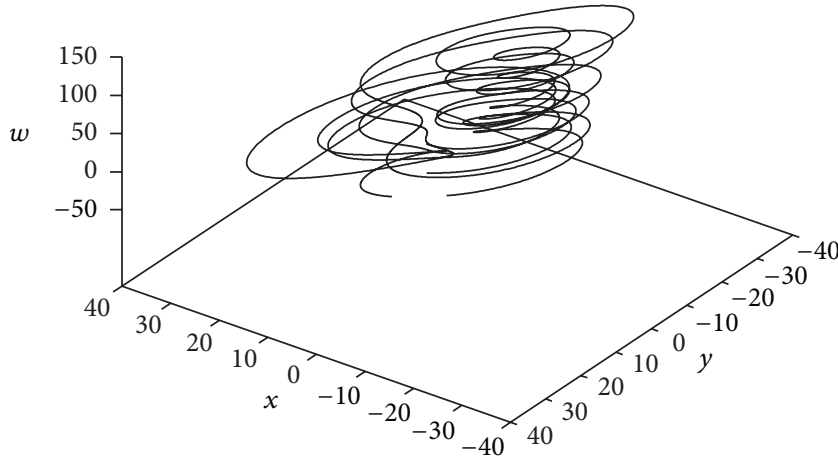

(c)

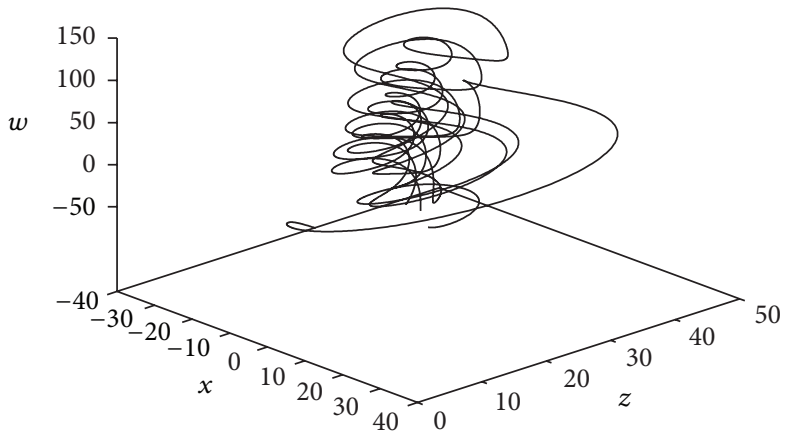

(b)

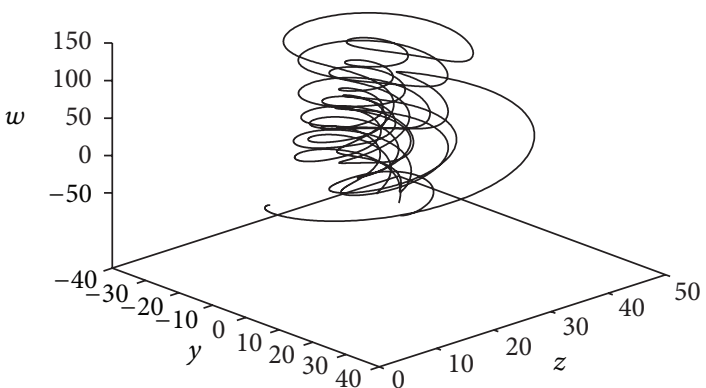

(d)

FIGURE 8: Phase portraits using 15-term MHPM on $\Delta t=0.001$ of Case 3 hyperchaotic Lü system.

$$
\begin{aligned}
& -1992411.473 t^{5}+11530028.33 t^{6} \\
& -71262877.62 t^{7}+718577249 t^{8} \\
& -6395732124 t^{9}+37269441880 t^{10} \\
& -159312883900 t^{11}+920702963800 t^{12} \\
& -6711925749000 t^{13}+13167072910000 t^{14} \\
y(t)= & 10.0-168.0 t-142.5000000 t^{2} \\
& +12667.65000 t^{3}-73101.80125 t^{4} \\
& -65386.07542 t^{5}-2385697.388 t^{6} \\
& +91130738.51 t^{7}-911565222 t^{8} \\
& +4123184965 t^{9}-11948855070 t^{10} \\
z(t)= & 23.0+91.0 t-2465.500000 t^{2} \\
& +152360063000 t^{11}-1552715738000 t^{12} \\
& +15732.00000 t^{3}-17025.01250 t^{4} \\
& +28954.84770 t^{5}-5141176.272 t^{6} \\
& \\
&
\end{aligned}
$$

$$
\begin{aligned}
& +31690639.8 t^{7}+354060035.5 t^{8} \\
& -6387494367 t^{9}+45947037200 t^{10} \\
& -321956587500 t^{11}+4065348055000 t^{12} \\
& -47403957200000 t^{13}+374722649800000 t^{14} \\
w(t)= & 19.0+392.7 t-1282.245000 t^{2} \\
& -14173.28950 t^{3}+131833.7322 t^{4} \\
& -192744.3592 t^{5}+2125982.525 t^{6} \\
& -97552191.45 t^{7}+1123539837 t^{8} \\
& -5986596371 t^{9}+19416967470 t^{10} \\
& -171790521600 t^{11}+1788038537000 t^{12} \\
& -636266254000 t^{13}-186275340000000 t^{14}
\end{aligned}
$$

4.2.2. Case 2. Subject to initial conditions, $\left(x_{0}, y_{0}, z_{0}, w_{0}\right)=$ $(5,8,-1,-3)$, (Figures 5 and 6$)$ the 15 -term solutions obtained by applying HPM on the chaotic Lü system are

$$
\begin{aligned}
x(t)= & 5.0+105.0 t+1075.550000 t^{2} \\
& +6249.805000 t^{3}+18225.35746 t^{4}
\end{aligned}
$$




$$
\begin{aligned}
& -138902.6781 t^{5}-2355709.162 t^{6} \\
& -23162852.18 t^{7}-154618310 t^{8} \\
& -748195754.8 t^{9}-1041804261 t^{10} \\
& +24770718260 t^{11}+356218971500 t^{12} \\
& +3051066581000 t^{13}+18921856670000 t^{14} \\
y(t)= & 8.0+165.0 t+1595.000000 t^{2} \\
& +8206.850000 t^{3}-2292.294583 t^{4} \\
& -546772.9699 t^{5}-7006911.000 t^{6} \\
& -58622123.15 t^{7}-347425673.6 t^{8} \\
& -1046027901 t^{9}+6772156980 t^{10} \\
& +1472886699200 t^{11}+1493057221000 t^{12} \\
& +10644960290000 t^{13}+51013501670000 t^{14}, \\
z(t)= & -1.0+43.0 t+768.00000 t^{2} \\
& +10533.46667 t^{3}+101093.2600 t^{4} \\
& +687900.1864 t^{5}+2608837.117 t^{6} \\
& -9163094.788 t^{7}-281215859.6 t^{8} \\
& -3111135091 t^{9}-23664129690 t^{10} \\
& -123625683400 t^{11}-222335906400 t^{12} \\
& +4216549901000 t^{13}+66291301000000 t^{14}, \\
& +303914658.0 t^{9}-8824703875 t^{10} \\
& +3.0-8.9 t+49.2150000 t^{2} \\
& +2447.809833 t^{3}+44122.08279 t^{4} \\
& +39587276.15 t^{7}+207303298.1 t^{8} \\
&
\end{aligned}
$$

4.2.3. Case 3. Subject to initial conditions, $\left(x_{0}, y_{0}, z_{0}, w_{0}\right)=$ $(6,0.5,4,-9)$, (Figures 7 and 8$)$ the 15 -term solutions obtained by applying HPM on the chaotic Lü system are

$$
\begin{aligned}
x(t)= & 6.0-207.0 t+3480.150000 t^{2} \\
& -38294.13500 t^{3}+381029.1811 t^{4} \\
& -2130472.098 t^{5}+12586769.73 t^{6}
\end{aligned}
$$

$$
\begin{aligned}
& -74222877.38 t^{7}+509799695.9 t^{8} \\
& -4201467628 t^{9}+36924472910 t^{10} \\
& -308119057600 t^{11}+2272132174000 t^{12} \\
& -13620775400000 t^{13}+49698306210000 t^{14} \\
y(t)= & 0.5-14.0 t+301.0000000 t^{2} \\
& -3094.033333 t^{3}+23158.95583 t^{4} \\
& -35982.41442 t^{5}-1895493.457 t^{6} \\
& +40327289.84 t^{7}-558681684.3 t^{8} \\
& +6261120403 t^{9}-59188221560 t^{10} \\
& +464930111600 t^{11}-2742488159000 t^{12} \\
& +5980055994000 t^{13}+139014056200000 t^{14} \\
z(t)= & 4.0-9.0 t-80.25000000 t^{2} \\
& +2228.275000 t^{3}-38856.29812 t^{4} \\
& +527729.0320 t^{5}-5734144.131 t^{6} \\
& +49172082.8 t^{7}-295481618.6 t^{8} \\
& +334170322.1 t^{9}+23383675250 t^{10} \\
& -453110248600 t^{11}+5896957194000 t^{12} \\
& -63041936150000 t^{13}+581842031400000 t^{14} \\
& -9.0+12.3 t-433.0050000 t^{2} \\
& +4913.064500 t^{3}-37032.37654 t^{4} \\
& +118989.8198 t^{5}+1801333.203 t^{6} \\
& -45408452.12 t^{7}+652121035.5 t^{8} \\
& -5408439997 t^{9}+70747365160 t^{10} \\
&
\end{aligned}
$$

\section{Conclusion}

In this work, the chaotic and hyperchaotic Lü systems are solved accurately by using the MHPM algorithm. The method has the advantage of giving an analytical form of the solution within each time interval which is not possible in purely numerical techniques like RK4. The present technique offers an explicit time-marching algorithm that works accurately over larger time steps than the RK4 method. The results presented in this paper suggest that MHPM is also readily 
applicable to hyperchaotic systems involving more complex dynamical behaviours.

\section{Conflict of Interests}

The authors declare that there is no conflict of interests regarding the publication of this paper.

\section{Acknowledgments}

The authors would like to acknowledge the financial support received from the Ministry of Higher Education via the RACE Grant no. RACE12-005-0005, International Islamic University Malaysia, and Universiti Putra Malaysia.

\section{References}

[1] E. N. Lorenz, "Deterministic non-periodic flow," Journal of the Atmospheric Sciences, vol. 20, pp. 130-141, 1963.

[2] R. Genesio and A. Tesi, "Harmonic balance methods for the analysis of chaotic dynamics in nonlinear systems," Automatica, vol. 28, no. 3, pp. 531-548, 1992.

[3] G. Chen and T. Ueta, "Yet another chaotic attractor," International Journal of Bifurcation and Chaos, vol. 9, no. 7, pp. 14651466, 1999.

[4] J. Lü and G. Chen, "A new chaotic attractor coined," International Journal of Bifurcation and Chaos in Applied Sciences and Engineering, vol. 12, no. 3, pp. 659-661, 2002.

[5] G. Pérez and H. A. Cerdeira, "Extracting messages masked by chaos," Physical Review Letters, vol. 74, no. 11, pp. 1970-1973, 1995.

[6] O. E. Rossler, "An equation for hyperchaos," Physics Letters A, vol. 71, no. 2-3, pp. 155-157, 1979.

[7] G. M. Mahmoud and M. E. Ahmed, "A hyperchaotic complex system generating two-, three-, and four-scroll attractors," Journal of Vibration and Control, vol. 18, no. 6, pp. 841-849, 2012.

[8] Y. Li, W. K. S. Tang, and G. Chen, "Generating hyperchaos via state feedback control," International Journal of Bifurcation and Chaos in Applied Sciences and Engineering, vol. 15, no. 10, pp. 3367-3375, 2005.

[9] A. Chen, J. Lu, J. Lü, and S. Yu, "Generating hyperchaotic Lü attractor via state feedback control," Physica A: Statistical Mechanics and its Applications, vol. 364, pp. 103-110, 2006.

[10] Q. Jia, "Hyperchaos generated from the Lorenz chaotic system and its control," Physics Letters A, vol. 366, no. 3, pp. 217-222, 2007.

[11] J. Lü and G. Chen, "A new chaotic attractor coined," International Journal of Bifurcation and Chaos, vol. 12, no. 3, pp. 659661, 2002.

[12] A. Vaněček and S. Čelikovské, Control Systems: From Linear Analysis to Synthesis of Chaos, Prentice-Hall, London, UK, 1996.

[13] Y. Yongguang and Z. Suochun, "Controlling uncertain Lü system using backstepping design," Chaos, Solitons and Fractals, vol. 15, no. 5, pp. 897-902, 2003.

[14] J. Lü, G. Chen, and S. Zhang, "Dynamical analysis of a new chaotic attractor," International Journal of Bifurcation and Chaos in Applied Sciences and Engineering, vol. 12, no. 5, pp. 1001-1015, 2002.

[15] A. Chen, J. Lu, J. Lü, and S. Yu, "Generating hyperchaotic Lü attractor via state feedback control," Physica A, vol. 364, pp. 103110, 2006.
[16] S. Ghosh, A. Roy, and D. Roy, "An adaptation of Adomian decomposition for numeric-analytic integration of strongly nonlinear and chaotic oscillators," Computer Methods in Applied Mechanics and Engineering, vol. 196, no. 4-6, pp. 1133-1153, 2007.

[17] G. Adomian, Nonlinear Stochastic Systems Theory and Applications to Physics, Kluwer Academic, New York, NY, USA, 1988.

[18] N. I. Razali, M. S. H. Chowdhury, and W. Asrar, "The multistage adomian decomposition method for solving chaotic lü system," Middle East Journal of Scientific Research, vol. 13, pp. 43-49, 2013.

[19] J.-H. He, "Variational iteration method-a kind of non-linear analytical technique: some examples," International Journal of Non-Linear Mechanics, vol. 34, no. 4, pp. 699-708, 1999.

[20] J.-H. He, "Variational iteration method for autonomous ordinary differential systems," Applied Mathematics and Computation, vol. 114, no. 2-3, pp. 115-123, 2000.

[21] S. M. Goh, M. S. M. Noorani, and I. Hashim, "Efficacy of variational iteration method for chaotic Genesio systemclassical and multistage approach," Chaos, Solitons and Fractals, vol. 40, no. 5, pp. 2152-2159, 2009.

[22] I. Hashim, M. S. M. Noorani, R. Ahmad, S. A. Bakar, E. S. Ismail, and A. M. Zakaria, "Accuracy of the adomian decomposition method applied to the Lorenz system," Chaos, Solitons \& Fractals, vol. 28, no. 5, pp. 1149-1158, 2006.

[23] M. M. Al-Sawalha, M. S. M. Noorani, and I. Hashim, "On accuracy of Adomian decomposition method for hyperchaotic Rössler system," Chaos, Solitons \& Fractals, vol. 40, no. 4, pp. 1801-1807, 2009.

[24] J. H. He, "A new approach to nonlinear partial differential equations," Communications in Nonlinear Science and Numerical Simulation, vol. 2, no. 4, pp. 230-235, 1997.

[25] J.-H. He, "Approximate analytical solution for seepage flow with fractional derivatives in porous media," Computer Methods in Applied Mechanics and Engineering, vol. 167, no. 1-2, pp. 57-68, 1998.

[26] J.-H. He, "Homotopy perturbation technique," Computer Methods in Applied Mechanics and Engineering, vol. 178, no. 3-4, pp. 257-262, 1999.

[27] J.-H. He, "A simple perturbation approach to Blasius equation," Applied Mathematics and Computation, vol. 140, no. 2-3, pp. 217-222, 2003.

[28] J.-H. He, "The homotopy perturbation method nonlinear oscillators with discontinuities," Applied Mathematics and Computation, vol. 151, no. 1, pp. 287-292, 2004.

[29] J.-H. He, "Homotopy perturbation method for solving boundary value problems," Physics Letters A, vol. 350, no. 1-2, pp. 87$88,2006$.

[30] J.-H. He, "Asymptotic methods for solitary solutions and compactons," Abstract and Applied Analysis, vol. 2012, Article ID 916793, 130 pages, 2012.

[31] M. Dehghan and F. Shakeri, "Use of he's homotopy perturbation method for solving a partial differential equation arising in modeling of flow in porous media," Journal of Porous Media, vol. 11, no. 8, pp. 765-778, 2008.

[32] A. Saadatmandi, M. Dehghan, and A. Eftekhari, "Application of He's homotopy perturbation method for non-linear system of second-order boundary value problems," Nonlinear Analysis. Real World Applications, vol. 10, no. 3, pp. 1912-1922, 2009.

[33] M. Rafei, D. D. Ganji, and H. Daniali, "Solution of the epidemic model by homotopy perturbation method," Applied Mathematics and Computation, vol. 187, no. 2, pp. 1056-1062, 2007. 
[34] M. S. Chowdhury and I. Hashim, "Solutions of Emden-Fowler equations by homotopy-perturbation method," Nonlinear Analysis: Real World Applications, vol. 10, no. 1, pp. 104-115, 2009.

[35] J. Biazar, B. Ghanbari, M. G. Porshokouhi, and M. G. Porshokouhi, "He's homotopy perturbation method: a strongly promising method for solving non-linear systems of the mixed Volterra-Fredholm integral equations," Computers \& Mathematics with Applications, vol. 61, no. 4, pp. 1016-1023, 2011.

[36] H. Saffari, I. Mansouri, M. H. Bagheripour, and H. Dehghani, "Elasto-plastic analysis of steel plane frames using homotopy perturbation method," Journal of Constructional Steel Research, vol. 70, pp. 350-357, 2012.

[37] M. S. Chowdhury, I. Hashim, S. Momani, and M. M. Rahman, "Application of multistage homotopy perturbation method to the chaotic Genesio system," Abstract and Applied Analysis, vol. 2012, Article ID 974293, 10 pages, 2012.

[38] Y. Khan and Z. ZdeněkŠmarda, "Heat transfer analysis on the Hiemenz flow of a non-Newtonian fluid: a homotopy method solution," Abstract and Applied Analysis, vol. 2013, Article ID 342690, 5 pages, 2013.

[39] Y. Khan, N. Faraz, A. Yildirim, and Q. Wu, "A series solution of the long porous slider," Tribology Transactions, vol. 54, no. 2, pp. 187-191, 2011.

[40] H. Jafari, K. Bagherian, and P. S. Moshokoa, "Homotopy perturbation method to obtain positive solutions of nonlinear boundary value problems of fractional order," Abstract and Applied Analysis, vol. 2014, Article ID 919052, 5 pages, 2014.

[41] M. S. H. Chowdhury, N. I. Razali, S. Ali, and M. M. Rahman, "An accurate analytic solution for differential and integral equations by modified homotopy perturbation method," Middle East Journal of Scientific Research, vol. 13, pp. 50-58, 2013.

[42] N. Herişanu and V. Marinca, "Optimal homotopy perturbation method for a non-conservative dynamical system of a rotating electrical machine," Zeitschrift für Naturforschung A, vol. 67, pp. 509-516, 2012.

[43] D. Olvera and A. Elías-Zúñiga, "Enhanced multistage homotopy perturbation method: approximate solutions of nonlinear dynamic systems," Abstract and Applied Analysis, vol. 2014, Article ID 486509, 12 pages, 2014.

[44] M. S. Chowdhury and I. Hashim, "Application of multistage homotopy-perturbation method for the solutions of the Chen system," Nonlinear Analysis. Real World Applications, vol. 10, no. 1, pp. 381-391, 2009.

[45] M. S. H. Chowdhury, I. Hashim, and S. Momani, "The multistage homotopy-perturbation method: a powerful scheme for handling the Lorenz system," Chaos, Solitons and Fractals, vol. 40, no. 4, pp. 1929-1937, 2009.

[46] I. Hashim and M. S. Chowdhury, "Adaptation of homotopyperturbation method for numeric-analytic solution of system of ODEs," Physics Letters: A, vol. 372, no. 4, pp. 470-481, 2008.

[47] I. Hashim, M. S. H. Chowdhury, and S. Mawa, "On multistage homotopy-perturbation method applied to nonlinear biochemical reaction model," Chaos, Solitons and Fractals, vol. 36, no. 4, pp. 823-827, 2008.

[48] M. S. H. Chowdhury, N. I. Razali, S. Ali, and M. M. Rahman, "A new application of multistage homotopy perturbation method to the Chaotic Rössler system," in Proceedings of the 2nd International Conference on Fundamental and Applied Sciences (ICFAS '12), vol. 1482 of AIP Conference Proceedings, pp. 507-511, Kuala Lumpur, Malaysia, June 2012. 


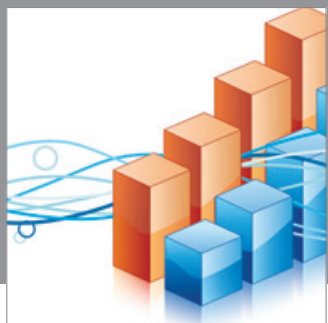

Advances in

Operations Research

mansans

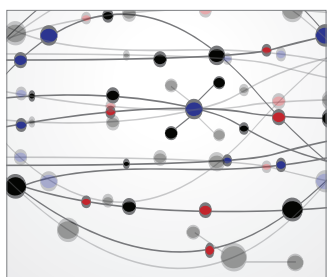

The Scientific World Journal
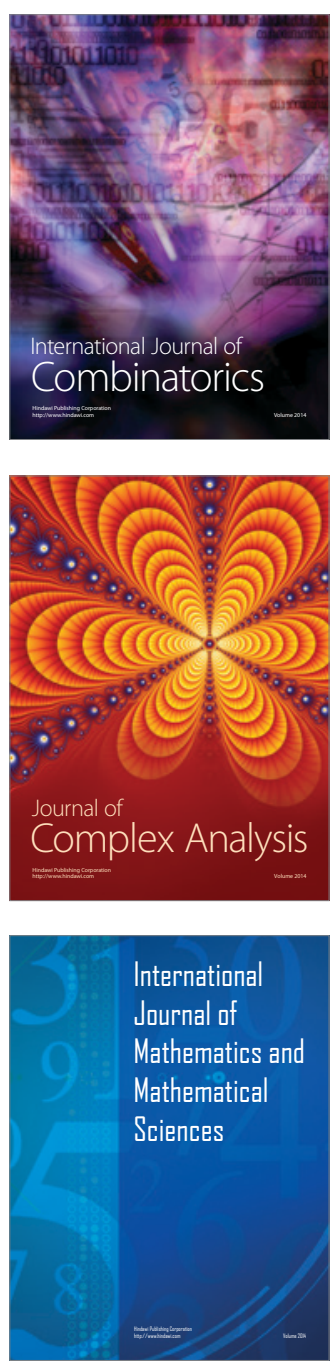
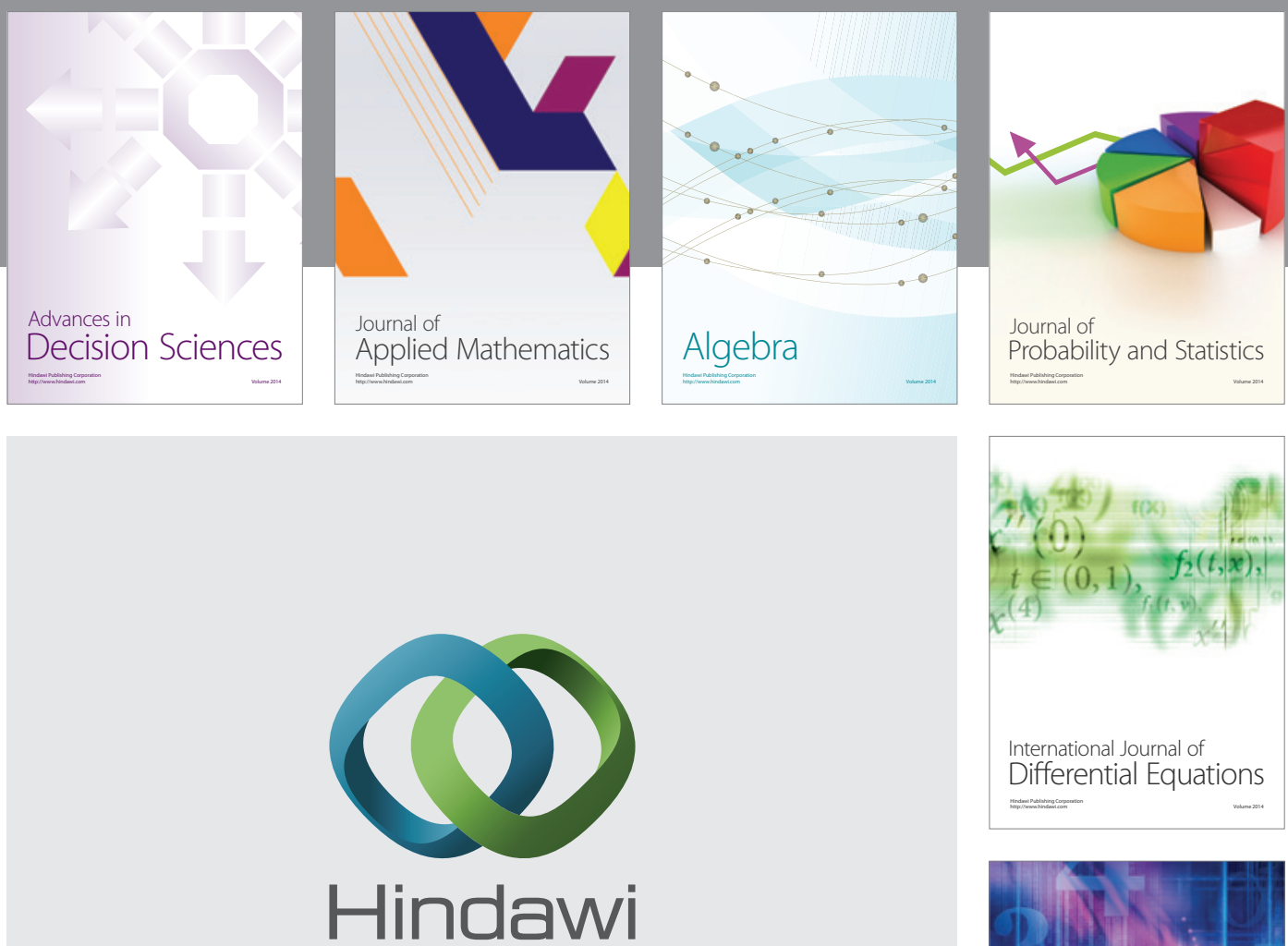

Submit your manuscripts at http://www.hindawi.com
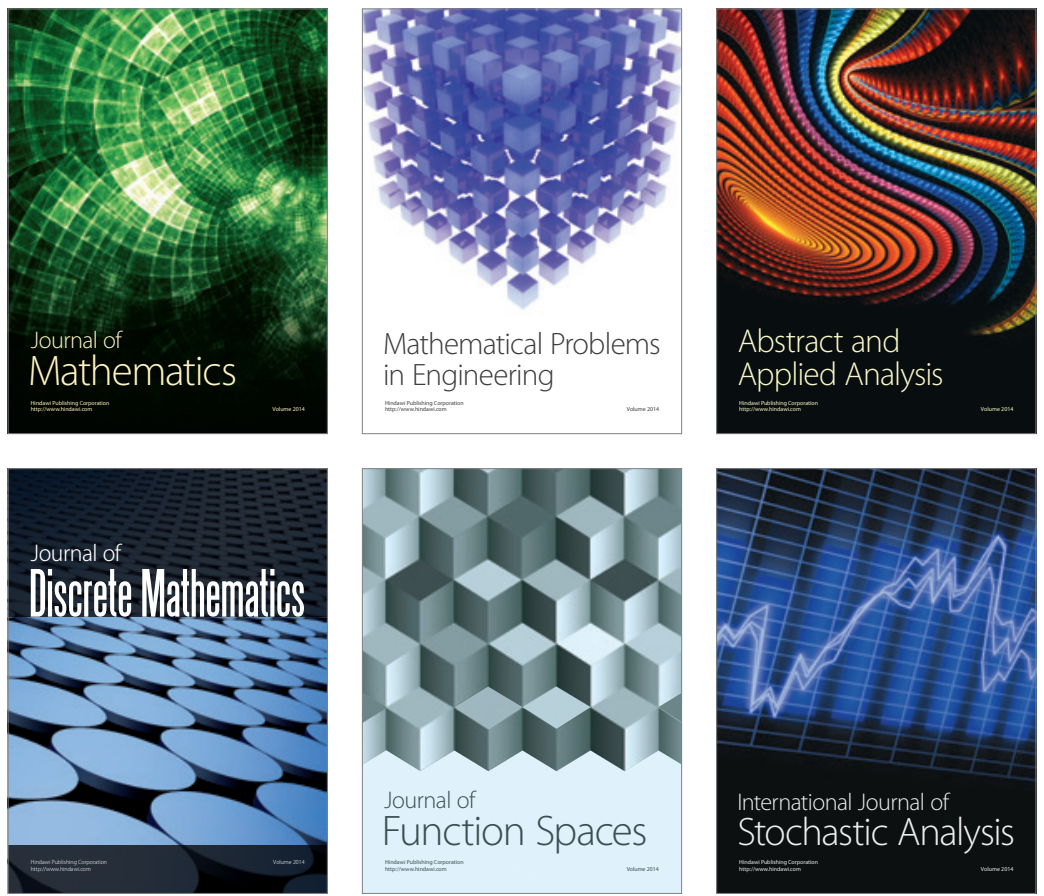

Journal of

Function Spaces

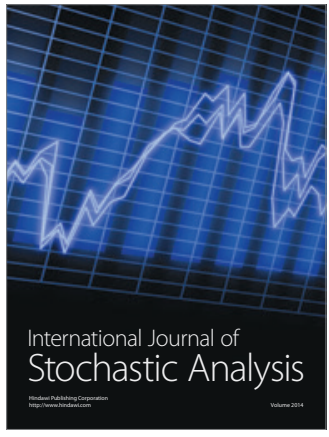

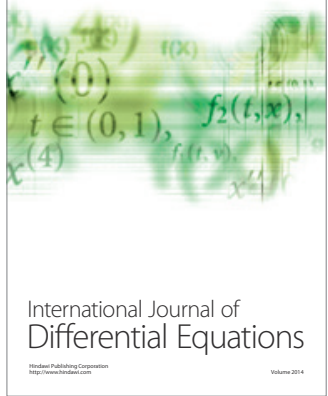
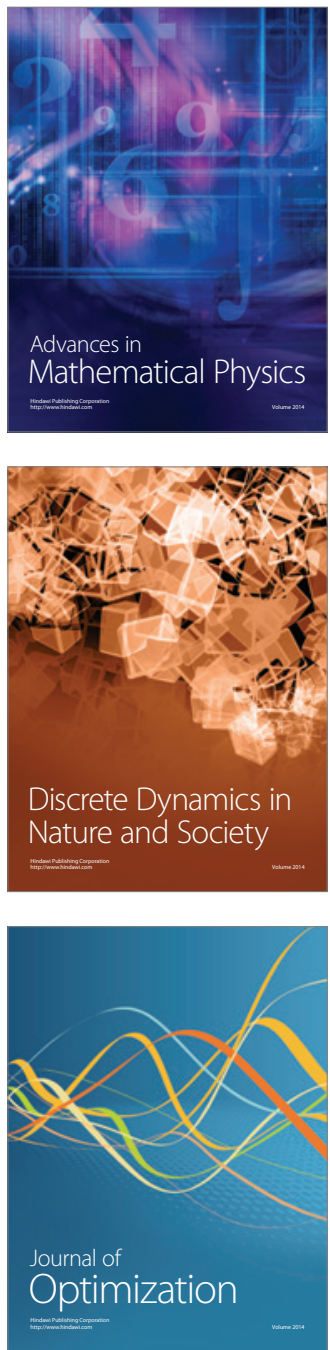